\title{
48. LABORATORY-DETERMINED SOUND VELOCITY, POROSITY, WET-BULK DENSITY, ACOUSTIC IMPEDANCE, ACOUSTIC ANISOTROPY, AND REFLECTION COEFFICIENTS FOR CRETACEOUS-JURASSIC TURBIDITE SEQUENCES AT DEEP SEA DRILLING PROJECT SITES 370 AND 416 OFF THE COAST OF MOROCCO ${ }^{1}$
}

\author{
Robert E. Boyce, Deep Sea Drilling Project, Scripps Institution of Oceanography, La Jolla, California
}

\begin{abstract}
From 661 to $880 \mathrm{~m}$ beneath the seafloor at DSDP Sites 370 and 416 are Albian to Barremian claystone with some limestone, sandstone, and siltstone. Compressional-wave velocities ranged from 1.70 to $4.37 \mathrm{~km} / \mathrm{s}$, with an average in situ vertical velocity of $1.93 \mathrm{~km} / \mathrm{s}$.

From 880 to $1430 \mathrm{~m}$ are Hauterivian to Valanginian turbidites of alternating graded calcareous and quartzose cycles from siltstone or fine sandstone to mudstone. Compressional-wave velocities range from 1.80 to $4.96 \mathrm{~km} / \mathrm{s}$ with an average in situ velocity of $2.61 \mathrm{~km} / \mathrm{s}$.

From 1430 to $1624 \mathrm{~m}$ are early Valanginian to Tithonian (Kimmeridgian?) turbidites, with alternating quartzose siltstone grading to mudstone cycles with hard micritic limestone and calcarenite (calciturbidites). Compressional-wave velocities range from 2.26 to $5.7 \mathrm{~km} / \mathrm{s}$, with an average in situ vertical velocity of $3.25 \mathrm{~km} / \mathrm{s}$.

Acoustic anisotropy is 0 to $30 \%$ faster parallel to bedding in Cretaceous to Tithonian sandstone-siltstone turbidites in mudstone and minor limestone from 661 to $1624 \mathrm{~m}$ below the seafloor. Between $2.0(?) \mathrm{km} / \mathrm{s}$ and $4.2(?) \mathrm{km} / \mathrm{s}$, anisotropy becomes particularly significant (below $1178 \mathrm{~m}$ ), where the anisotropy is about $+0.4 \mathrm{~km} / \mathrm{s}$ or greater. The mudstone, softer sandstone, and softer siltstone tend to have velocities around 2.0 to $2.5 \mathrm{~km} / \mathrm{s}$; the cemented sandstone and limestone cluster around $2.5 \mathrm{~km} / \mathrm{s}$ to $4.2 \mathrm{~km} / \mathrm{s}$; thus the relative percentage anisotropy is greater for lower-velocity lithologies. Above $4.2(?) \mathrm{km} / \mathrm{s}$, the well-cemented sandstone and limestone tend to have a smaller (less than $+0.4 \mathrm{~km} / \mathrm{s}$ ) absolute anisotropy, and many samples are nearly isotropic.

These physical property data are separated into depth plots for (1) mudstone, (2) siltstone (3) sandstone, (4) marlstone, and (5) limestone. The mudstone's porosity and wet-bulk density curves versus depth are slightly higher and lower, respectively, than similar porosity and wet-bulk density curves summarized in Hamilton (1976). These differences could be some combination of (1) differences in laboratory methods; (2) age, lithologic, and cementation differences; or (3) overconsolidation created by a geological sequence which has been eroded away.

If the average in situ vertical velocities calculated by Boyce (1980b) for Hole 416A are correct, then the 1.43-s (round-trip) reflector (blue) discussed by Lancelot and Winterer (1980) and Lancelot, Winterer et al. (1980b), would correlate to about $1500 \mathrm{~m}$ in Hole $416 \mathrm{~A}$, and not below $1624 \mathrm{~m}$ as suggested by Lancelot and Winterer. There appears to be a significant change in the acoustic character at or around that depth $(1500 \mathrm{~m})$ to a more lithified, calcareous, and cemented lithology. This does not prove Lancelot and Winterer (1980) and Lancelot, Winterer et al. (1980b) to be incorrect, but only suggests another possible interpretation.
\end{abstract}

\section{INTRODUCTION}

This chapter is concerned with the physical property relationships enumerated below, using data measured on cores recovered from Sites 370 and 416, in the Atlantic Ocean off the coast of Morocco (Fig. 1) for the Cretaceous-Tithonian sandstone, siltstone, mudstone, and minor micritic limestone and calcarenite from 661 to $1624 \mathrm{~m}$ below the seafloor. The objectives of the chapter are as follows:

1) At Site 416 , to study physical properties versus depth for different lithologic types: (a) mudstone, (b) siltstone, (c) sandstone, (d) marlstone, and (e) limestone. This is important for characterizing the lithology and physical properties of this area and for making comparisons to other areas.

2) At Sites 370 and 416 , to undertake additional systematic studies of the horizontal and vertical velocity, acoustic anisotropy, porosity, and wet-bulk density of

\footnotetext{
${ }^{1}$ Hay, w. W., Sibuet, J.-C., et al., Init. Repts. DSDP, 75: Washington (U.S. Govt. Printing Office).
}

the geologic sequence. This information is valuable for correctly interpreting gravity data, seismic reflection data, seismic refraction data, and sonobuoy data.

3) At Sites 370 and 416, to attempt to calculate reflection coefficients; and by using in situ interval velocities calculated by Boyce (1980b) for the geologic section penetrated at Site 416, to attempt to correlate the acoustic character of the data obtained from Sites 370 and 416 with the 1.43-s (round-trip) reflector (blue) in seismic profiles discussed by Lancelot, Winterer, et al. (1980b) and Lancelot and Winterer (1980).

\section{DATA, DEFINITIONS, AND METHODS}

For Site 416 data, the sediment classification is discussed in Lancelot, Winterer, et al. (1980a), and detailed age-dates and lithologies are discussed in Lancelot, Winterer, et al. (1980b). Wet-bulk density is defined as the ratio of weight of water-saturated sediment or rock sample to its volume, expressed as $\mathrm{g} / \mathrm{cm}^{3}$. Wet-water content is the ratio of the weight of seawater in the sample to the weight of the saturated sample, and is expressed as a percentage. Porosity is the ratio of the pore volume in a sample to the volume of the saturated sam- 


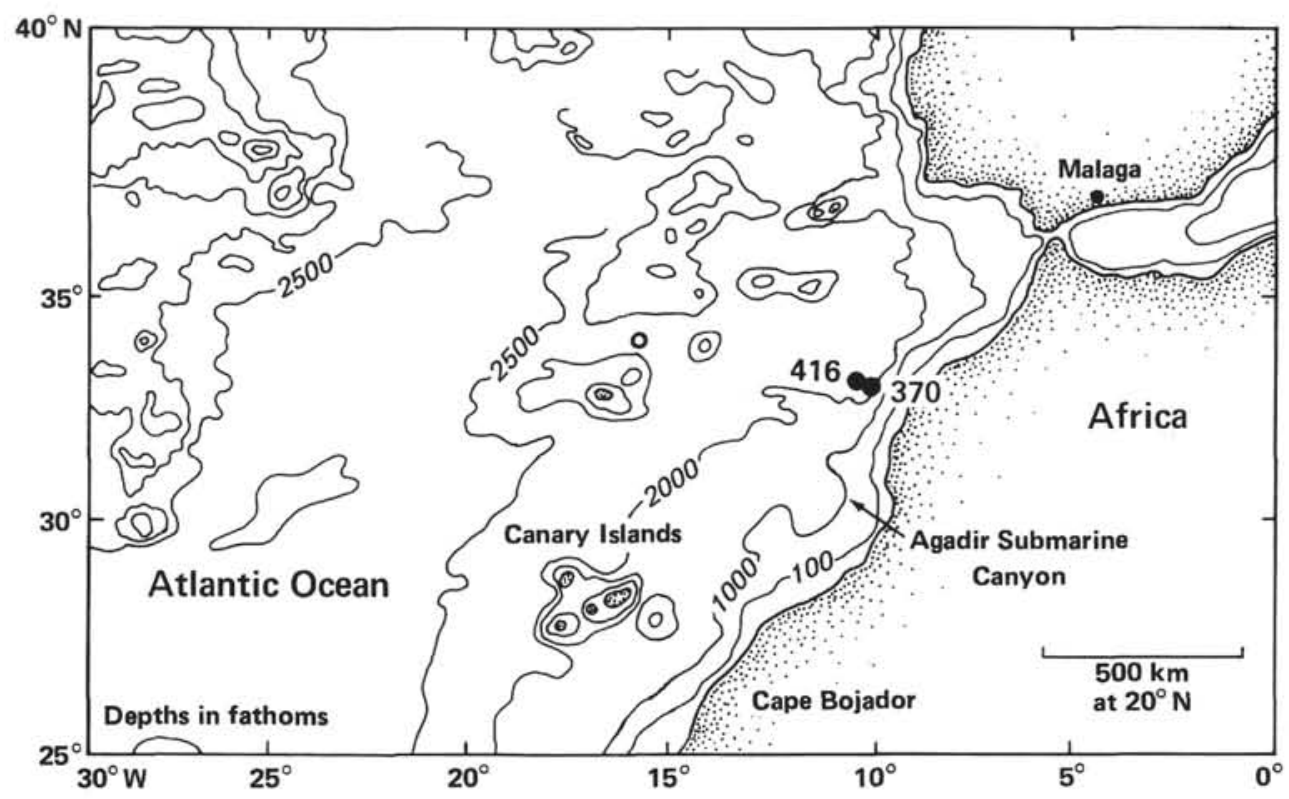

Figure 1. Index map showing locations of DSDP Sites 370 and 416.

ple and is also expressed as a percentage. Acoustic impedance is defined as the product of the velocity and wet-bulk density, and is expressed as $\left(\mathrm{g} \cdot 10^{5}\right) /\left(\mathrm{cm}^{2} \bullet \mathrm{s}\right)$. All the equations, derivations, and techniques are discussed in detail in Boyce (1980a).

With respect to sampling at Site 416 , we generally waited at least $4 \mathrm{hr}$. after the core had been brought on deck to allow it to reach room temperature. We then cut and removed an undisturbed (visible, undistorted bedding), water-saturated, compressional-sound-velocity sample, about $2.5 \mathrm{~cm}$ thick. The sample was carefully smoothed with a sharp knife or file. Velocities were measured to within $\pm 2 \%$ accuracy with the Hamilton Frame velocimeter (Boyce, 1980a), perpendicular and parallel to bedding. Immediately afterward, its wet-bulk density was measured to within \pm 2 or $3 \%$ precision with the Gamma Ray Attenuation Porosity Evaluator (GRAPE) (Evans, 1965) as modified in Boyce (1980a), using special 2-minute gamma-ray counts. Then, the wet-water content of a subsample was determined by weighing the sample both wet and after drying $24 \mathrm{hr}$. at $110^{\circ} \mathrm{C}$. The weight of evaporated water was corrected for salt $(45 \%)$ ) to give the weight of seawater (Boyce, 1980a; Hamilton, 1971b). The estimated precision is $\pm 2.5 \%$ (absolute). Porosity (precision of $\pm 6 \%$ ) is determined from the product of the wet-water content and wet-bulk density, divided by the density of the interstitial water $\left(1.032 \mathrm{~g} / \mathrm{cm}^{3}\right)$. The acoustic impedance is obtained from the product of the vertical velocity and the wet-bulk density. The laboratory results of Site 416 Cretaceous-Tithonian sedimentary rocks have been tabulated in Table 1.

All data for Site 370 are from Table 1 in Trabant (1978) and will not be tabulated here. Trabant (1978) and Lancelot et al. (1978) discuss their methods and the sediment classification used at Site 370. Detailed litholo- gies and age-dates for Site $\mathbf{3 7 0}$ data are discussed in detail in Lancelot, Seibold, et al. (1978).

Compressional-wave (sound) velocity in isotropic material has been defined (Wood, 1941; Bullen, 1947; Birch, 1961; Hamilton, 1971a, b) as

$$
V=\left(\frac{\varkappa+4 \mu / 3}{\varrho_{b}}\right)^{1 / 2}
$$

where $V$ is the compressional-wave velocity; $\varrho_{b}$ is the wet-bulk density in $\mathrm{g} / \mathrm{cm}^{3}$ and $\varrho_{b}=\varrho_{w} \phi+(1-\phi) \varrho_{g}$ (here $\phi$ is the fractional porosity of the sediment or rock, and the subscripts $b, g$, and $w$ represent the wetbulk density, grain density, and water density, respectively); $\varkappa$ is the incompressibility or bulk modulus; and $\mu$ is the shear (rigidity) modulus

Where samples are anisotropic, $x$ and $\mu$ may have various unique values for the corresponding vertical and horizontal directions. See Laughton (1957), Carlson and Christensen (1977), Gregory (1977), and Bachman (1979) for a discussion of anisotropy.

Compressional-wave (sound) velocity of sediments and rocks has been related to sediment components by Wood (1941), Wyllie et al. (1956), Nafe and Drake (1957) and others. Relationships of the Site 416 data to these equations are discussed in Boyce (1980b). Velocity is related to mineralogical composition, fluid content, water saturation of pores, temperature, pressure, grain size, texture, cementation, direction with respect to bedding or foliation, and alteration, as summarized by Press (1966). Recently, Hamilton $(1976,1978,1980)$ has summarized the velocity-density relationships of sediment and rock of the seafloor for oceanic sediments and sedimentary rock.

Acoustic impedance is the product of the vertical velocity and wet-bulk density. Reflection coefficients 
Table 1. Data on physical properties, Hole 416A.

\begin{tabular}{|c|c|c|c|c|c|c|c|}
\hline & & & Compre & sional-sou & d velocity & & $\begin{array}{r}\text { GRAPE } \\
\text { wet-bulk } \\
2 \text {-min. } \\
(\mathrm{g} / \mathrm{cr}\end{array}$ \\
\hline & Depth in & & 1 & Anis & tropy & & \\
\hline $\begin{array}{l}\text { Core-Section } \\
\text { (interval in cm) }\end{array}$ & $\begin{array}{l}\text { hole } \\
(\mathrm{m})\end{array}$ & $\begin{array}{c}\text { Beds } \\
(\mathrm{km} / \mathrm{s})\end{array}$ & $\begin{array}{c}\text { Beds } \\
(\mathrm{km} / \mathrm{s})\end{array}$ & $\underset{(\mathrm{km} / \mathrm{s})}{1-1}$ & $\begin{array}{c}(1-1) / \perp \\
(\%)\end{array}$ & $\begin{array}{l}\text { Temp. } \\
\left({ }^{\circ} \mathrm{C}\right)\end{array}$ & $\underset{\text { Beds }}{1}$ \\
\hline $5-1,30-32$ & 754.30 & & 1.717 & & & 25 & \\
\hline 1, 56-58 & 754.56 & 3.861 & 3.466 & 0.395 & 11.40 & 26 & \\
\hline $6-1,23-25$ & 891.23 & 1.886 & 1.767 & 0.119 & 6.73 & 25 & \\
\hline $2,18-20$ & 892.68 & 1.897 & 1.767 & 0.130 & 7.36 & 26 & \\
\hline 2. $140-150$ & 893.95 & & & & & & \\
\hline $3,16-18$ & 894.16 & 1.868 & 1.805 & 0.063 & 3.49 & 26 & 2.100 \\
\hline $7-1,33-35$ & 991.83 & 4.666 & 4.451 & 0.215 & 4.83 & 25 & \\
\hline $2,12-14$ & 993.12 & 1.988 & 1.836 & 0.152 & 8.28 & 26 & 2.269 \\
\hline 2. $140-150$ & 994.45 & & & & & & \\
\hline $3,2-4$ & 994.52 & 2.003 & 1.801 & 0.202 & 11.22 & 25 & 2.152 \\
\hline CC, $14-15$ & & & & & & & 2.600 \\
\hline $8-7,9-11$ & 1102.09 & 2.588 & 2.631 & -0.043 & -1.63 & 26 & \\
\hline $9-1,146-148$ & 1178.36 & 2.421 & 1.858 & 0.563 & 30.30 & 27 & \\
\hline $2,58-60$ & 1178.98 & 4.325 & 3.887 & 0.438 & 11.27 & 27 & \\
\hline $3,145-147$ & 1181.35 & 2.489 & 2.398 & 0.091 & 3.79 & 27 & \\
\hline $4,125-127$ & 1182.65 & 2.330 & 2.176 & 0.154 & 7.08 & 27 & \\
\hline $4,140-150$ & 1182.85 & & & & & & \\
\hline $6,11-13$ & 1184.51 & 4.536 & 4.526 & 0.010 & 2.21 & 27 & \\
\hline $10-1,13-16$ & 1185.53 & 4.553 & 4.602 & -0.049 & -1.06 & 27 & \\
\hline $2,6-8$ & 1186.96 & 2.623 & 2.133 & 0.490 & 22.97 & 27 & \\
\hline $2,30-32$ & 1187.20 & 3.014 & 2.591 & 0.423 & 16.33 & 27 & \\
\hline $11-1,2-4$ & 1194.82 & 4.947 & 4.422 & 0.525 & 11.87 & 26 & \\
\hline $1,9-11$ & 1194.89 & 2.607 & 2.162 & 0.445 & 20.58 & 26.5 & \\
\hline $2,89-91$ & 1197.19 & 2.497 & 2.207 & 0.290 & 13.14 & 26 & \\
\hline $4,140-150$ & 1200.75 & & & & & & \\
\hline $5,135-137$ & 1202.15 & 2.502 & 2.085 & 0.417 & 20.00 & 26 & \\
\hline $6,77-79$ & 1203.07 & 2.357 & 2.130 & 0.227 & 10.66 & 26 & \\
\hline $12-1,10-12$ & 1204.40 & 4.836 & 4.686 & 0.150 & 3.20 & 25 & \\
\hline $2,42-44$ & 1206.22 & 2.602 & 2.175 & 0.427 & 19.63 & 25 & \\
\hline $3,29-31$ & 1207.59 & 3.083 & 2.489 & 0.594 & 23.87 & 25 & \\
\hline $3,86-87$ & 1208.17 & & & & & & \\
\hline $4,42-44$ & 1209.22 & 2.738 & 2.287 & 0.451 & 19.72 & 25 & \\
\hline $13-1,7-9$ & 1213.87 & 2.386 & 2.019 & 0.367 & 18.17 & 26 & \\
\hline $2,42-44$ & 1215.72 & 4.432 & 4.545 & -0.113 & -2.49 & 26 & \\
\hline $3,28-30$ & 1217.08 & 2.274 & 2.076 & 0.198 & 9.54 & 25 & \\
\hline $14-1,5-7$ & 1223.45 & 2.460 & 2.065 & 0.395 & 19.13 & 26 & \\
\hline $2,49-51$ & 1225.39 & 2.604 & 2.216 & 0.388 & 17.51 & 26 & \\
\hline $3,96-98$ & 1227.36 & 3.883 & 4.069 & -0.186 & -4.57 & 25 & \\
\hline $4,62-64$ & 1228.52 & 4.322 & 3.545 & 0.777 & 21.92 & 25 & \\
\hline $5,69-70$ & 1230.09 & 2.376 & 2.240 & 0.136 & 6.07 & 25 & \\
\hline $15-1,2-4$ & 1232.92 & 2.316 & 2.083 & 0.233 & 11.19 & 25 & \\
\hline $2,11-13$ & 1234.51 & 2.454 & 2.011 & 0.443 & 22.03 & 25 & \\
\hline $3,0-2$ & 1235.90 & 4.585 & 3.848 & 0.737 & 19.15 & 25 & \\
\hline $4,7-8$ & 1237.47 & 2.421 & 2.291 & 0.130 & 5.67 & 25 & \\
\hline $5,133-135$ & 1240.23 & 2.721 & 2.341 & 0.380 & 16.23 & 25 & \\
\hline $6,55-57$ & 1240.95 & 2.128 & 2.006 & 0.122 & 6.08 & 25 & \\
\hline $16-1,2-4$ & 1242.52 & 4.532 & 4.432 & 0.100 & 2.26 & 25 & \\
\hline $2,6-8$ & 1244.06 & 2.175 & 2.001 & 0.174 & 8.70 & 25 & \\
\hline $3,95-97$ & 1246.45 & 2.698 & 2.191 & 0.507 & 23.14 & 25 & \\
\hline $4,7-8$ & 1247.07 & 2.434 & 2.174 & 0.260 & 11.96 & 25 & \\
\hline $17-1,33-35$ & 1252.33 & 2.416 & 2.019 & 0.397 & 19.66 & 24 & \\
\hline $1,86-88$ & 1252.86 & 2.039 & 1.989 & 0.050 & 2.51 & 25 & \\
\hline $1,98-100$ & 1252.98 & 2.592 & 2.136 & 0.456 & 21.35 & 25 & \\
\hline $2,52-54$ & 1254.02 & 2.335 & 2.075 & 0.260 & 12.53 & 25 & \\
\hline $4,28-30$ & 1256.78 & 3.688 & 3.249 & 0.439 & 13.51 & 25 & \\
\hline $18-1,49-50$ & 1261.99 & 2.140 & 2.044 & 0.096 & 4.70 & 25 & \\
\hline $2,25-27$ & 1263.25 & 4.613 & 3.855 & 0.758 & 19.66 & 27 & \\
\hline $3,3-5$ & 1264.53 & 2.493 & 2.083 & 0.410 & 19.68 & 27 & \\
\hline $4,2-4$ & 1266.02 & 2.711 & 2.295 & 0.416 & 18.13 & 27 & \\
\hline $4,66-68$ & 1266.66 & 2.539 & 2.177 & 0.362 & 16.63 & 25 & \\
\hline $19-1,15-17$ & 1271.15 & 2.509 & 2.082 & 0.427 & 20.51 & 25 & \\
\hline $2,31-33$ & 1272.81 & 2.081 & 2.004 & 0.077 & 3.84 & 25 & \\
\hline $3,11-13$ & 1274.11 & 4.090 & 3.531 & 0.559 & 15.83 & 25 & \\
\hline $3,36-38$ & 1274.36 & 2.307 & 2.111 & 0.196 & 9.28 & 25 & \\
\hline CC, $18-20$ & 1278.24 & 4.181 & 3.734 & 0.447 & 11.97 & 25 & \\
\hline $20-1,6-8$ & 1277.56 & 2.649 & 2.233 & 0.416 & 18.63 & 25 & \\
\hline $1,40-43$ & 1277.90 & 3.267 & 3.259 & 0.008 & 0.25 & 25 & 2.439 \\
\hline $2,59-61$ & 1279.59 & 2.351 & 2.160 & 0.191 & 8.84 & 25 & \\
\hline $2,70-72$ & 1279.70 & 2.483 & 2.109 & 0.374 & 17.73 & 25 & \\
\hline 2. $95-97$ & 1279.95 & 2.947 & 2.540 & 0.407 & 16.02 & 25 & \\
\hline $21-1,57-59$ & 1290.27 & 4.289 & 3.880 & 0.409 & 10.54 & 25 & \\
\hline $2,103-105$ & 1292.53 & 2.599 & 2.249 & 0.350 & 15.56 & 25 & 2.341 \\
\hline $3,88-90$ & 1293.88 & 2.813 & 2.360 & 0.453 & 19.19 & 25 & \\
\hline $4,46-48$ & 1294.96 & 3.412 & 3.136 & 0.276 & 8.80 & 25 & \\
\hline $5,4-6$ & 1296.04 & 2.660 & 1.819 & 0.841 & $46.23 ?$ & 25 & \\
\hline $22-1,0-2$ & 1299.50 & 2.473 & 2.051 & 0.422 & 20.58 & 25 & \\
\hline $2,22-24$ & 1301.22 & 2.774 & 2.358 & 0.416 & 17.64 & 25 & \\
\hline $3,8-10$ & 1302.58 & 3.709 & 3.036 & 0.673 & 22.17 & 26 & \\
\hline $4,52-54$ & 1304.52 & 2.278 & & & & 26 & \\
\hline $4,57-59$ & 1304.57 & 4.446 & 4.044 & 0.402 & 9.94 & 26 & \\
\hline $23-1,2-4$ & 1309.12 & 2.555 & 2.121 & 0.434 & 20.46 & 26 & \\
\hline $2,5-7$ & 1310.65 & 2.473 & 2.053 & 0.420 & 20.46 & 26 & 2.320 \\
\hline $3,54-55$ & 1312.64 & 2.699 & 2.305 & 0.394 & 17.09 & 26 & \\
\hline $4,13-15$ & 1313.73 & 2.791 & 2.380 & 0.411 & 17.27 & 26 & \\
\hline $5,22-24$ & 1315.32 & 3.612 & 3.010 & 0.602 & 20.00 & 26 & \\
\hline $24-1,3-5$ & 1318.63 & 2.336 & 2.036 & 0.300 & 14.73 & 25 & \\
\hline 1. $95-97$ & 1319.55 & 2.730 & 2.375 & 0.355 & 14.95 & 25 & \\
\hline $2,0-2$ & 1320.10 & 2.224 & 2.049 & 0.175 & 8.54 & 25 & \\
\hline
\end{tabular}


Table 1. (Continued).

\begin{tabular}{|c|c|c|c|c|c|c|c|c|}
\hline & & & Compre & sional-sou & d velocity & & $\begin{array}{r}\text { GRAPE } \\
\text { wet-bulk } \\
2-\mathrm{min} . \\
(\mathrm{g} / \mathrm{c}\end{array}$ & $\begin{array}{l}\text { "special" } \\
\text { density } \\
\text { count } \\
\left.\mathrm{m}^{3}\right)^{b} \\
\end{array}$ \\
\hline $\begin{array}{c}\text { Core-Section } \\
\text { (interval in cm) }\end{array}$ & $\begin{array}{l}\text { Depth in } \\
\text { hole } \\
\text { (m) }\end{array}$ & $\begin{array}{c}\text { I } \\
\text { Beds } \\
(\mathrm{km} / \mathrm{s})\end{array}$ & $\begin{array}{c}\perp \\
\text { Beds } \\
(\mathrm{km} / \mathrm{s})\end{array}$ & $\begin{array}{l}\text { Anis } \\
1-1 \\
(\mathrm{~km} / \mathrm{s})\end{array}$ & $\begin{array}{l}\left(\mid-\frac{1}{)}\right) / 1 \\
(\%)\end{array}$ & $\begin{array}{l}\text { Temp. } \\
\left({ }^{\circ} \mathrm{C}\right)\end{array}$ & $\begin{array}{c}1 \\
\text { Beds }\end{array}$ & $\stackrel{1}{\text { Beds }}$ \\
\hline $2,123-129$ & 1321.33 & 4.531 & 3.373 & 1.158 & 34.33 & 25 & & 2.635 \\
\hline $3,96-98$ & 1322.59 & 2.402 & 2.026 & 0.376 & 18.56 & 25 & & \\
\hline $25-1,94-96$ & 1328.65 & 4.478 & 4.091 & 0.387 & 9.46 & 25 & & 2.616 \\
\hline $2,16-18$ & 1329.36 & 3.459 & 3.021 & 0.438 & 14.50 & 25 & & 2.240 \\
\hline $2,123-125$ & 1330.43 & 2.406 & 2.246 & 0.160 & 7.12 & 25 & & 2.286 \\
\hline $3,20-22$ & 1330.90 & 2.468 & 2.460 & 0.008 & 0.33 & 25 & & 2.414 \\
\hline $4,3-5$ & 1332.23 & 2.317 & 2.084 & 0.233 & 11.18 & 25 & & 2.254 \\
\hline $5,113-115$ & 1334.83 & 2.515 & 2.044 & 0.471 & 23.04 & 25 & & \\
\hline $26-1,72-74$ & & & & & & & & 2.379 \\
\hline $2,3-4$ & 1338.73 & 2.362 & 2.077 & 0.285 & 13.72 & 25 & & 2.220 \\
\hline $3,34-36$ & 1340.54 & 2.254 & 2.209 & 0.045 & 2.04 & 25 & & 2.287 \\
\hline $4,32-34$ & 1342.02 & 2.097 & & & & 25 & 2.228 & \\
\hline $5,55-57$ & 1343.75 & 2.809 & 2.422 & 0.387 & 15.98 & 25 & & 2.382 \\
\hline $27-1,0-2$ & 1346.70 & 2.657 & 2.274 & 0.383 & 16.84 & 26 & & 2.519 \\
\hline $2,39-41$ & 1348.59 & 4.615 & 4.362 & 0.253 & 5.80 & 26 & & 2.666 \\
\hline $3,11-13$ & 1349.81 & 3.856 & 2.925 & 0.931 & 31.83 & 26 & & 2.419 \\
\hline $3,106-107$ & 1350.76 & 2.887 & 2.466 & 0.421 & 17.07 & 26 & & 2.373 \\
\hline $28-1,12-14$ & 1356.32 & 2.874 & 2.462 & 0.412 & 16.73 & 25 & & 2.172 \\
\hline $2,59-61$ & 1358.29 & 4.376 & 3.672 & 0.704 & 19.17 & 25 & & 2.529 \\
\hline $3,85-87$ & 1360.05 & 2.423 & 2.221 & 0.202 & 9.10 & 25 & & 2.408 \\
\hline $5,10-12$ & 1362.30 & 2.493 & 2.196 & 0.297 & 13.52 & 25 & & 2.361 \\
\hline $6,2-4$ & 1363.72 & 2.501 & 2.256 & 0.245 & 10.86 & 25 & & 2.393 \\
\hline $29-1,52-54$ & 1366.22 & 4.307 & 3.852 & 0.455 & 11.81 & 25 & & 2.593 \\
\hline $2,2-4$ & 1367.22 & 2.518 & 2.088 & 0.430 & 20.59 & 25 & & 2.300 \\
\hline 3, $97-99$ & 1369.67 & 2.823 & 2.384 & 0.439 & 18.41 & 25 & & 2.365 \\
\hline $4,103-105$ & 1371.23 & 2.433 & 3.349 & -0.916 & -27.35 & 25 & & 2.326 \\
\hline $5,51-53$ & 1372.21 & 2.193 & 2.127 & 0.066 & 3.10 & 25 & & 2.299 \\
\hline $6,127-129$ & 1374.47 & 2.791 & 2.335 & 0.456 & 19.53 & 25 & & 2,408 \\
\hline $30-1,77-79$ & 1375.97 & 2.715 & 2.310 & 0.405 & 17.53 & 25 & & 2.277 \\
\hline $2,107-109$ & 1377.77 & 3.239 & 2.672 & 0.567 & 21.22 & 25 & & \\
\hline $3,29-31$ & 1379.49 & 2.655 & 2.472 & 0.183 & 7.40 & 25 & & 2.368 \\
\hline $4,147-149$ & 1381.17 & 2.681 & 2.259 & 0.422 & 18.68 & 25 & & 2.191 \\
\hline $5,104-106$ & 1382.24 & 2.497 & 2.240 & 0.257 & 11.47 & 25 & & 2.292 \\
\hline $31-1,14-16$ & 1384.74 & 3.242 & 2.814 & 0.428 & 15.21 & 25 & & 2.436 \\
\hline $2,81-82$ & 1386.91 & 2.643 & 2.269 & 0.374 & 16.48 & 25 & & 2.323 \\
\hline $2,144-146$ & 1387.54 & 4.189 & 4.289 & -0.100 & -2.33 & 25 & & 2.574 \\
\hline $3,35-37$ & 1387.95 & 2.626 & 2.358 & 0.268 & 11.37 & 25 & & 2.406 \\
\hline $3,95-97$ & 1388.55 & 3.142 & 2.513 & 0.629 & 25.03 & 25 & 2.462 & 2.361 \\
\hline $32-1,21-23$ & 1394.31 & 3.067 & & & & 25 & & 2.398 \\
\hline 1. $131-133$ & 1395.41 & 2.572 & 2.112 & 0.460 & 21.78 & 25 & & 2.313 \\
\hline $2,0-2$ & 1395.60 & 2.984 & 2.490 & 0.494 & 19.84 & 25 & & 2.352 \\
\hline $2,100-104$ & 1396.60 & 3.778 & 3.438 & 0.340 & 9.89 & 25 & & 2.334 \\
\hline $3,71-74$ & 1397.81 & 3.053 & 2.475 & 0.578 & 23.35 & 25 & & 2.386 \\
\hline $34-1,12-14$ & 1406.92 & 3.144 & 2.683 & 0.461 & 17.18 & 26 & & 2.410 \\
\hline $2,6-8$ & 1408.36 & 2.724 & 2.270 & 0.454 & 20.00 & 26 & & 2.364 \\
\hline $3,33-35$ & 1410.13 & 2.766 & 2.544 & 0.222 & 8.73 & 26 & & 2.406 \\
\hline $4,24-26$ & 1411.54 & 4.594 & 4.303 & 0.291 & 6.76 & 26 & & 2.591 \\
\hline $35-1,35-39$ & 1416.75 & 3.357 & 2.771 & 0.586 & 21.15 & 26 & & 2.431 \\
\hline $1,58-60$ & 1416.98 & 2.975 & 2.262 & 0.713 & 31.52 & 26 & & 2.335 \\
\hline $2,82-84$ & 1418.72 & 2.930 & 2.440 & 0.490 & 20.08 & 26 & & 2.331 \\
\hline $3,0-2$ & 1419.40 & 5.257 & 4.293 & 0.964 & 22.46 & 26 & & 2.556 \\
\hline $36-1,16-18$ & 1425.56 & 3.820 & 2.994 & 0.826 & 27.59 & 26 & & 2.521 \\
\hline $1,130-133$ & 1426.70 & 3.099 & 2.458 & 0.641 & 26.08 & 25 & 2.435 & \\
\hline $2,68-70$ & 1427.58 & 3.244 & 2.766 & 0.478 & 17.28 & 25 & & 2.439 \\
\hline $3,8-10$ & 1428.48 & 3.428 & 3.014 & 0.414 & 13.74 & 25 & & 2.514 \\
\hline 3, $29-31$ & 1428.69 & 3.456 & 3.077 & 0.379 & 12.32 & 25 & & 2.501 \\
\hline $37-2,2-4$ & 1436.52 & 3.204 & 2.749 & 0.455 & 16.55 & 25 & & 2.491 \\
\hline $2,54-57$ & 1437.04 & 2.761 & 2.215 & 0.546 & 24.65 & 25 & & 2.336 \\
\hline $3,27-29$ & 1438.27 & 4.326 & 3.569 & 0.757 & 21.21 & 25 & & 2.550 \\
\hline $4,17-19$ & 1439.67 & 2.803 & 2.332 & 0.471 & 20.20 & 25 & & 2.403 \\
\hline $4,35-37$ & 1439.85 & 3.785 & 3.003 & 0.782 & 26.04 & 25 & & 2.509 \\
\hline $4,55-57$ & 1440.05 & 3.882 & 3.580 & 0.302 & 8.44 & 25 & & 2.483 \\
\hline 4. $70-72$ & 1440.20 & 3.356 & 2.976 & 0.380 & 12.77 & 25 & & 2.457 \\
\hline $38-1,10-12$ & 1445.10 & 3.180 & 2.113 & 1.067 & 50.50 & 25 & & 2.447 \\
\hline $1,44-46$ & 1445.44 & 3.277 & 2.754 & 0.523 & 18.99 & 25 & & 2.463 \\
\hline $1,135-138$ & 1446.35 & 2.836 & 2.368 & 0.468 & 19.76 & 25 & & 2.411 \\
\hline $2,38-41$ & 1446.88 & 4.300 & 3.822 & 0.478 & 12.51 & 25 & & 2.576 \\
\hline $2,44-46$ & 1446.94 & 3.277 & 2.887 & 0.390 & 13.51 & 25 & & 2.473 \\
\hline $40-1,33-36$ & 1454.83 & 3.162 & 2.494 & 0.668 & 26.78 & 24 & & 2.234 \\
\hline 1. $80-82$ & 1455.30 & 3.999 & 3.386 & 0.613 & 18.10 & 24 & & 2.558 \\
\hline $2,127-128$ & 1457.27 & 3.702 & 3.291 & 0.411 & 12.49 & 24 & & 2.534 \\
\hline $3,23-25$ & 1457.73 & 3.580 & 2.699 & 0.881 & 32.64 & 24 & & 2.414 \\
\hline $3,48-50$ & 1457.98 & 4.705 & 4.402 & 0.303 & 6.88 & 24 & & 2.650 \\
\hline $3,79-81$ & 1458.29 & 4.460 & 3.466 & 0.994 & 28.68 & 24 & & 2.548 \\
\hline $41-1,4-5$ & 1463.94 & 4.532 & 4.474 & 0.058 & 1.30 & 26 & & 2.643 \\
\hline $1,84-87$ & 1464.74 & 3.205 & 2.394 & 0.811 & 33.88 & 26 & & 2.264 \\
\hline $3,107-109$ & 1467.97 & 4.692 & 4.401 & 0.291 & 6.61 & 26 & & 2.586 \\
\hline $4,110-113$ & 1469.50 & 3.373 & 2.909 & 0.464 & 15.95 & 26 & & 2.524 \\
\hline $5,8-9$ & 1469.98 & 4.635 & 4.038 & 0.597 & 14.78 & 26 & & 2.585 \\
\hline $42-1,43-45$ & 1473.73 & 5.161 & 5.484 & -0.323 & -5.89 & 27 & & 2.692 \\
\hline 1, 96-98 & 1474.26 & 3.951 & 2.747 & 1.204 & 43.83 & 26 & & 2.431 \\
\hline $2,110-113$ & 1475.90 & 3.882 & 3.648 & 0.234 & 6.41 & 25 & & 2.709 \\
\hline $3,58-60$ & 1476.88 & 3.737 & 3.432 & 0.305 & 8.89 & 26 & & 2.519 \\
\hline $43-1,35-37$ & 1482,75 & 3.702 & 3.338 & 0.364 & 10.90 & 26 & & 2.570 \\
\hline 1, $80-82$ & 1483.20 & 3.021 & 2.476 & 0.545 & 22.01 & 26 & 2.386 & \\
\hline $1,93-95$ & 1483.33 & 3.781 & 3.252 & 0.529 & 16.27 & 26 & & 2.534 \\
\hline $2,36-38$ & 1484.26 & 3.844 & 3.362 & 0.482 & 14.34 & 26 & & 2.481 \\
\hline $3,66-68$ & 1486.06 & 3.597 & 3.057 & 0.540 & 17.66 & 26 & & 2.398 \\
\hline
\end{tabular}


Table 1. (Continued).

\begin{tabular}{|c|c|c|c|c|c|c|c|c|c|c|c|c|c|}
\hline \multirow[b]{2}{*}{$\begin{array}{l}\text { Core-Section } \\
\text { (interval in cm) }\end{array}$} & \multirow[b]{2}{*}{$\begin{array}{l}\text { Depth in } \\
\text { hole } \\
\text { (m) }\end{array}$} & \multicolumn{5}{|c|}{ Compressional-sound velocity } & \multicolumn{2}{|c|}{$\begin{array}{c}\text { GRAPE "special" } \\
\text { wet-bulk density" } \\
\text { 2-min. count } \\
\left(\mathrm{g} / \mathrm{cm}^{3}\right)^{\mathrm{b}}\end{array}$} & \multirow{2}{*}{$\begin{array}{l}\text { Wet } \\
\text { water } \\
\text { content } \\
\text { Salt corr. } \\
(\%)\end{array}$} & \multirow[b]{2}{*}{$\begin{array}{c}\text { Porosity }{ }^{\mathrm{c}} \\
(\%)\end{array}$} & \multirow[b]{2}{*}{$\begin{array}{c}\text { Acoustic } \\
\text { impedançe } \\
\left(\mathrm{g} \cdot 10^{5} / \mathrm{cm}^{2} \cdot \mathrm{s}\right)\end{array}$} & \multirow[b]{2}{*}{ Lithology ${ }^{d}$} & \multirow[t]{2}{*}{. } \\
\hline & & $\begin{array}{c}\text { Beds } \\
(\mathrm{km} / \mathrm{s})\end{array}$ & $\begin{array}{c}\perp \\
\text { Beds } \\
(\mathrm{km} / \mathrm{s})\end{array}$ & $\begin{array}{c}\text { Anis } \\
1-1 \\
(\mathrm{~km} / \mathrm{s})\end{array}$ & $\begin{array}{l}\text { tropy } \\
\left(\mid-\frac{1}{1}\right) / \perp \\
(\%)\end{array}$ & $\begin{array}{l}\text { Temp. } \\
\left({ }^{\circ} \mathrm{C}\right)\end{array}$ & $\underset{\text { Beds }}{\text { I }}$ & $\stackrel{1}{\text { Beds }}$ & & & & & \\
\hline $44-1,72-74$ & 1492.62 & 3.660 & 3.066 & 0.594 & 19.37 & 26 & & 2.475 & 4.94 & 11.85 & 7.59 & Laminated sandstone (olv. gy.) & \\
\hline $1,89-91$ & 1492.79 & 3.188 & 2.437 & 0.751 & 30.82 & 25 & & 2.378 & 9.11 & 20.99 & 5.80 & Mudstone (dsky. y, brn.) & \\
\hline $1,143-145$ & 1493.33 & 4.095 & 3.751 & 0.344 & 9.17 & 25 & & 2.671 & 3.10 & 8.02 & 10.02 & Limestone (pale y. brn.) & \\
\hline $2,104-106$ & 1494.45 & 3.146 & 2.787 & 0.359 & 12.88 & 25 & & 2.416 & 8.17 & 19.13 & 6.73 & Laminated siltstone (dsky. y. brn.) & \\
\hline $45-1,13-15$ & 1501.33 & 5.163 & 4.730 & 0.433 & 9.15 & 25 & & 2.655 & 5.11 & 13.15 & 12.56 & Calcarenite (pale y. brn.) & \\
\hline $1,65-67$ & 1501.85 & 4.229 & 4.170 & 0.059 & 1.41 & 25 & & 2.498 & 3.99 & 9.66 & 10.42 & Laminated sandstone (gy. red) & \\
\hline $1,68-70$ & 1501.88 & 3.197 & 2.429 & 0.768 & 31.62 & 25 & & 2.283 & 6.30 & 13.94 & 5.55 & Mudstone (mod. brn.) & \\
\hline $2,72-75$ & 1503.42 & 5.050 & 5.165 & -0.115 & -2.23 & 25 & & 2.653 & 1.27 & 3.26 & 13.70 & Limestone (pale y. brn.) & \\
\hline $3,8-10$ & 1504.28 & 3.362 & $1.922 ?$ & 1.440 & 74.92 & 25 & & 2.519 & 7.94 & 19.38 & 4.84 & Laminated siltstone (dsky. y. brn.) & \\
\hline $46-1,6-8$ & 1510.66 & 3.451 & 2.966 & 0.485 & 16.35 & 26 & & 2.540 & 5.18 & 12.75 & 7.53 & Mudstone (mod. brn.) & \\
\hline $1,74-76$ & 1511.34 & 3.439 & 2.950 & 0.489 & 16.58 & 25 & & 2.509 & 2.97 & 7.22 & 7.40 & Marlstone (dk. y. brn.) & \\
\hline $1,82-84$ & 1511.42 & 4.434 & 4.211 & 0.223 & 5.30 & 26 & & 2.617 & 3.37 & 8.55 & 11.02 & Limestone (pale y. brn.) & \\
\hline $2,0-2$ & 1512.10 & 3.765 & 3.191 & 0.574 & 17.99 & 26 & & 2.437 & 3.42 & 8.08 & 7.78 & Laminated sandstone (dk. y. brn.) & \\
\hline $2,20-22$ & 1512.30 & 4.264 & 4.095 & 0.169 & 4.13 & 26 & & 2.647 & 0.63 & 1.62 & 10.84 & Limestone (gy, olv.) & \\
\hline $47-1,11-13$ & 1520.11 & 3.705 & 3.334 & 0.371 & 11.12 & 26 & & 2.545 & 3.38 & 8.34 & 8.49 & Laminated sandstone (dk. y. brn.) & \\
\hline $1,36-38$ & 1520.36 & 3.187 & 2.443 & 0.744 & 30.45 & 26 & & 2.408 & 9.17 & 21.40 & 5.88 & Mudstone (dsky. brn.) & \\
\hline $2,98-100$ & 1522.48 & 3.343 & 4.454 & -1.111 & -24.94 & 26 & & 2.614 & 3.48 & 8.81 & 11.64 & Limestone (pale y. brn.) & \\
\hline $48-1,6-9$ & 1529.56 & 2.776 & 2.245 & 0.531 & 23.65 & 25 & & 2.327 & 7.35 & 16.57 & 5.22 & Mudstone (mod. brn.) & \\
\hline $1,52-53$ & 1530.02 & 4.053 & 3.732 & 0.321 & 8.60 & 26 & & 2.572 & 2.58 & 6.43 & 9.60 & Marlstone (dk. y. brn.) & \\
\hline $1,97-100$ & 1530.47 & 4.605 & 4.803 & -0.198 & -4.12 & 26 & & 2.643 & 1.75 & 4.48 & 12.69 & Limestone (pale y. brn.) & \\
\hline $1,107-110$ & 1530.57 & 4.548 & 4.332 & 0.216 & 4.99 & 26 & & 2.520 & 1.12 & 2.73 & 10.92 & Laminated sandstone (olv. gy.) & \\
\hline $2,0-2$ & 1531.00 & 3.364 & 2.997 & 0.367 & 12.25 & 26 & & 2.465 & 7.17 & 17.13 & 7.39 & Laminated siltstone (olv. gy.) & \\
\hline $49-1,24-26$ & 1539.34 & 3.685 & 2.975 & 0.710 & 23.87 & 25 & & 2.474 & 5.53 & 13.26 & 7.36 & Laminated siltstone (dsky. y. brn.) & \\
\hline $1,43-46$ & 1539.53 & 3.413 & 2.623 & 0.790 & 30.12 & 25 & & 2.353 & 7.01 & 15.98 & 6.17 & Mudstone (gyish. brn.) & \\
\hline $2,18-20$ & 1540.78 & 4.878 & 4.545 & 0.333 & 7.33 & 25 & & 2.613 & 2.51 & 6.36 & 11.88 & Limestone (pale y. brn.) & \\
\hline $2,55-57$ & 1541.15 & 4.554 & 4.413 & 0.141 & 3.20 & 25 & & 2.674 & 2.31 & 5.99 & 11.80 & Calcarenite (dk. y. brn.) & \\
\hline $2,133-135$ & 1541.93 & 4.551 & 3.733 & 0.818 & 21.91 & 25 & & & 2.20 & & & Laminated sandstone (olv. gy.) & \\
\hline $50-1,6-8$ & 1548.66 & 2.987 & 2.465 & 0.522 & 21.18 & 26 & & 2.373 & 8.48 & 19.50 & 5.85 & Mudstone (gy. olv. grn.) & \\
\hline $1,18-20$ & 1548.78 & 3.998 & 3.640 & 0.358 & 9.84 & 26 & & 2.600 & 2.61 & 6.58 & 9.46 & Marlstone (mod. brn.) & \\
\hline $1,23-25$ & 1548.83 & 5.281 & 5.216 & 0.065 & 1.25 & 26 & & 2.610 & 2.17 & 5.49 & 13.61 & Calcarenite (pale y. brn.) & \\
\hline $1,133-135$ & 1549.93 & 4.623 & 4.392 & 0.231 & 5.26 & 26 & & 2.575 & 2.82 & 7.04 & 11.31 & Limestone (pale y. brn.) & \\
\hline $2,82-83$ & 1550.92 & 3.120 & 2.616 & 0.504 & 19.27 & 26 & & 2.282 & 7.57 & 16.74 & 5.97 & Laminated siltstone (dsky, y. brn.) & \\
\hline $3,18-20$ & 1551.78 & 4.441 & 4.160 & 0.281 & 6.75 & 26 & & 2.600 & 3.10 & 7.81 & 10.82 & Laminated sandstone (olv. gy.) & \\
\hline $51-1,24-25$ & 1558.24 & 3.998 & 3.643 & 0.355 & 9.74 & 26 & & 2.511 & 4.07 & 9.90 & 9.15 & Limestone (pale brn.) & \\
\hline $1,33-34$ & 1558.33 & 4.742 & 4.645 & 0.097 & 2.09 & 26 & & 2.629 & 3.42 & 8.71 & 12.21 & Laminated sandstone (lt. olv, gy.) & \\
\hline $2,24-25$ & 1559.74 & 3.136 & 2.490 & 0.646 & 25.94 & 26 & & 2.338 & 7.59 & 17.20 & 5.82 & Laminated mudstone (dsky. y. brn.) & \\
\hline $2,72-74$ & 1560.22 & 4.746 & 4.581 & 0.165 & 3.60 & 26 & & 2.613 & 1.92 & 4.86 & 11.97 & Calcarenite (gy. olv.) & \\
\hline $52-1,9-10$ & 1567.49 & 4.430 & 4.243 & 0.187 & 4.41 & 25 & & 2.630 & 1.85 & 4.71 & 11.16 & Limestone (lt. olv. gy.) & \\
\hline $1,31-35$ & 1567.71 & 3.135 & 2.357 & 0.778 & 33.01 & 25 & & 2.391 & 11.09 & 25.70 & 5.64 & Mudstone (gy, brn.) & \\
\hline $1,79-80$ & 1568.19 & 4.323 & 4.184 & 0.639 & 3.32 & 25 & & 2.545 & 1.64 & 4.04 & 10.65 & Calcarenite (tt. olv. gy.) & \\
\hline $2,96-98$ & 1569.86 & 4.809 & 4.786 & 0.023 & 0.48 & 25 & & & 2.39 & & & Laminated sandstone (olv. gy.) & \\
\hline $3,16-18$ & 1570.56 & 3.882 & 2.970 & 0.912 & 30.71 & 25 & & 2.478 & 4.98 & 11.96 & 7.36 & Laminated siltstone (mod. brn.) & \\
\hline $53-1,0-2$ & 1576.80 & 4.475 & 4.480 & -0.005 & -0.10 & 26 & & 2.539 & 0.83 & 2.04 & 11.37 & Laminated sandstone (gy, olv.) & \\
\hline $1,7-9$ & 1576.87 & 3.277 & 2.623 & 0.654 & 24.93 & 26 & & 2.435 & 5.81 & 13.71 & 6.38 & Mudstone (gy. brn.) & \\
\hline $1,107-109$ & 1577.87 & 3.417 & 3.064 & 0.353 & 11.52 & 26 & & 2.442 & 5.27 & 12.47 & 7.48 & Laminated siltstone (dsky. y. brn.) & \\
\hline $3,140-142$ & 1581.20 & 5.251 & 4.896 & 0.355 & 7.25 & 26 & & 2.681 & 2.75 & 7.14 & 13.13 & Limestone (pale y. brn.) & \\
\hline $57-1,11-13$ & 1614.61 & 3.307 & 2.528 & 0.779 & 30.81 & 25 & & 2.429 & 8.02 & 18.88 & 6.14 & Mudstone (dsky, brn.) & \\
\hline $1,36-38$ & 1614.86 & 4.358 & 3.966 & 0.392 & 9.88 & 26 & & 2.555 & 2.00 & 4.95 & 10.13 & Sandstone (tt, olv. gy.) & \\
\hline $1,44-45$ & 1614.94 & 4.955 & 4.510 & 0.445 & 9.87 & 26 & & 2.629 & 1.40 & 3.57 & 11.86 & Limestone (pale y, brn.) & \\
\hline $1,104-106$ & 1615.54 & 3.811 & 3.111 & 0.700 & 22.50 & 25 & & 2.415 & 3.12 & 7.30 & 7.51 & Laminated siltstone (dsky. y. brn.) & \\
\hline $1,145-147$ & 1615.95 & 5.699 & 5.532 & 0.167 & 3.02 & 25 & 2.717 & & 0.74 & 1.94 & $15.03^{\mathrm{e}}$ & Calcarenite (pale y. brn.) & \\
\hline
\end{tabular}

a The calculation used the following parametrs: $\varrho \mathrm{g}, \varrho_{\mathrm{gc}}=2.7 \mathrm{~g} / \mathrm{cm}^{3} ; \mathrm{ef}=1.025 \mathrm{~g} / \mathrm{cm}^{3}$. $\mathrm{efc}=1.128 \mathrm{~g} / \mathrm{cm}^{3}, \mu=0.1028 \mathrm{~cm}^{2} / \mathrm{g}$ for Cores 1 through 16 , and $\mu=0.1024 \mathrm{~cm} / \mathrm{g}$ for Cores 17

b $\mathrm{\text {through }}$ S.I. units are $\mathrm{m} / \mathrm{s}=(\mathrm{km} / \mathrm{s}) \times 1000 ; \mathrm{kg} / \mathrm{m}^{3}=\left(\mathrm{g} / \mathrm{cm}^{3}\right) / 10^{3}$.

c Porosity $=$ [salt corrected wet-water content $) \times($ wet-bulk density) $] /($ density interstitial water $)$; assume salinity $=45 \%$ and interstitial water density $=1.032 \mathrm{~g} / \mathrm{cm}^{3}$.

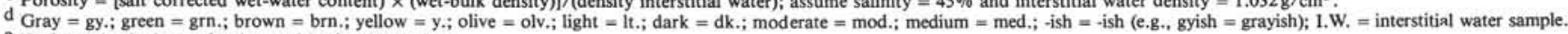

$\mathrm{e}^{\mathrm{G}}$ Horizontal velocity or density used in the calculation.

(R.C.) from 661 to $1624 \mathrm{~m}$ are calculated from the impedance data:

$$
\text { R.C. }=\frac{I_{1}-I_{0}}{I_{1}+I_{0}}
$$

where $I_{o}$ is the upper acoustic impedance and $I_{1}$ is the lower impedance value. Reflection coefficients are computed very simply by using the upper and lower impedance values as they are listed in their tables, and plotting the reflection coefficient value at the same depth as the lower impedance value. Because of this very simple approach, investigators must be very careful about precisely correlating these reflection coefficients to reflectors in the seismic profiles.

\section{RESULTS}

The physical property data discussed in this chapter are for the following Cretaceous-Tithonian geologic sequence at Site 370 and Site 416 (Lancelot, Winterer, et al., 1980b):

1) From 661 to $880 \mathrm{~m}$ below the seafloor are Albian to Barremian claystone with some limestone, sandstone, and siltstone.

2) From 880 to $1430 \mathrm{~m}$ are Hauterivian to Valanginian turbidites of alternating graded calcareous and quartzose cycles from siltstone or fine sandstone to mudstone.

3) From 1430 to $1624 \mathrm{~m}$ are early Valanginian to Tithonian (Kimmeridgian?) turbidites with alternating quartzose siltstone grading to mudstone cycles with hard micritic limestone and calcarenite (calciturbidites). 


\section{R. E. BOYCE}

For the Cretaceous-Tithonian geologic sequence at Sites 416 and 370 , as discussed above, horizontal compressional-wave velocity versus depth below the seafloor is plotted in Figure 2, and vertical compressional-wave velocity versus depth are shown in Figure 3. From Figure 3 there is an obvious change in acoustic character at $\sim 1375$ and $\sim 1475 \mathrm{~m}$.

From 1475 to $1624 \mathrm{~m}$ velocities are widely scattered; this is caused by varying amounts of cementation by calcium carbonate. Even the relatively lower-velocity mudstone, in general, appears to have a slightly higher velocity than similar relative low-velocity mudstone above $\sim 1475$ meters. The very high vertical velocities (greater than $5.0 \mathrm{~km} / \mathrm{s}$ ) are the micritic limestone, but particularly the well-cemented calcarenites below $\sim 1473$ meters.

The first (going down the hole) high vertical velocity ( $>5 \mathrm{~km} / \mathrm{s}$ ) layer occurs at $\sim 1474$ meters, but this is a relatively thin layer and thus it may not create a significant reflection on the seismic profile. However, it could be possible that an important reflector is created as a result of the overall increase in cementation or lithification of the entire lower geologic sequence from $\sim 1474$ meters and downward. If this is assumed to be possible, then the actual reflector in the seismic profiles may be lower, perhaps at approximately $1500 \mathrm{~m}$ or more. This would allow for the proper ideal thickness (about a quarter wave length) needed for a significant reflecting horizon.

Figure 4 displays reflection coefficients versus depth. It is not particularly helpful as there is such wide variation in all the data; thus it does not appear to be as useful as Figure 3 in helping to identify potential seismic reflectors. However, at about 1460 to $1500 \mathrm{~m}$ there are some variations in velocity which could be a potential reflector.

Acoustic anisotropy is important for estimating vertical velocities (for seismic-reflection profiles) from the horizontal velocities determined by refraction techniques, and the oblique velocities determined by sonobuoy techniques. Acoustic anisotropy in sedimentary rock may be created by some combination of the following variables as summarized in Press (1966): (1) alternating layers with high- or low-velocity materials; (2) tabular minerals that are aligned with bedding, thus creating fewer gaps in a direction parallel to bedding; (3) minerals with acoustic anisotropy, whose high-velocity axis may be

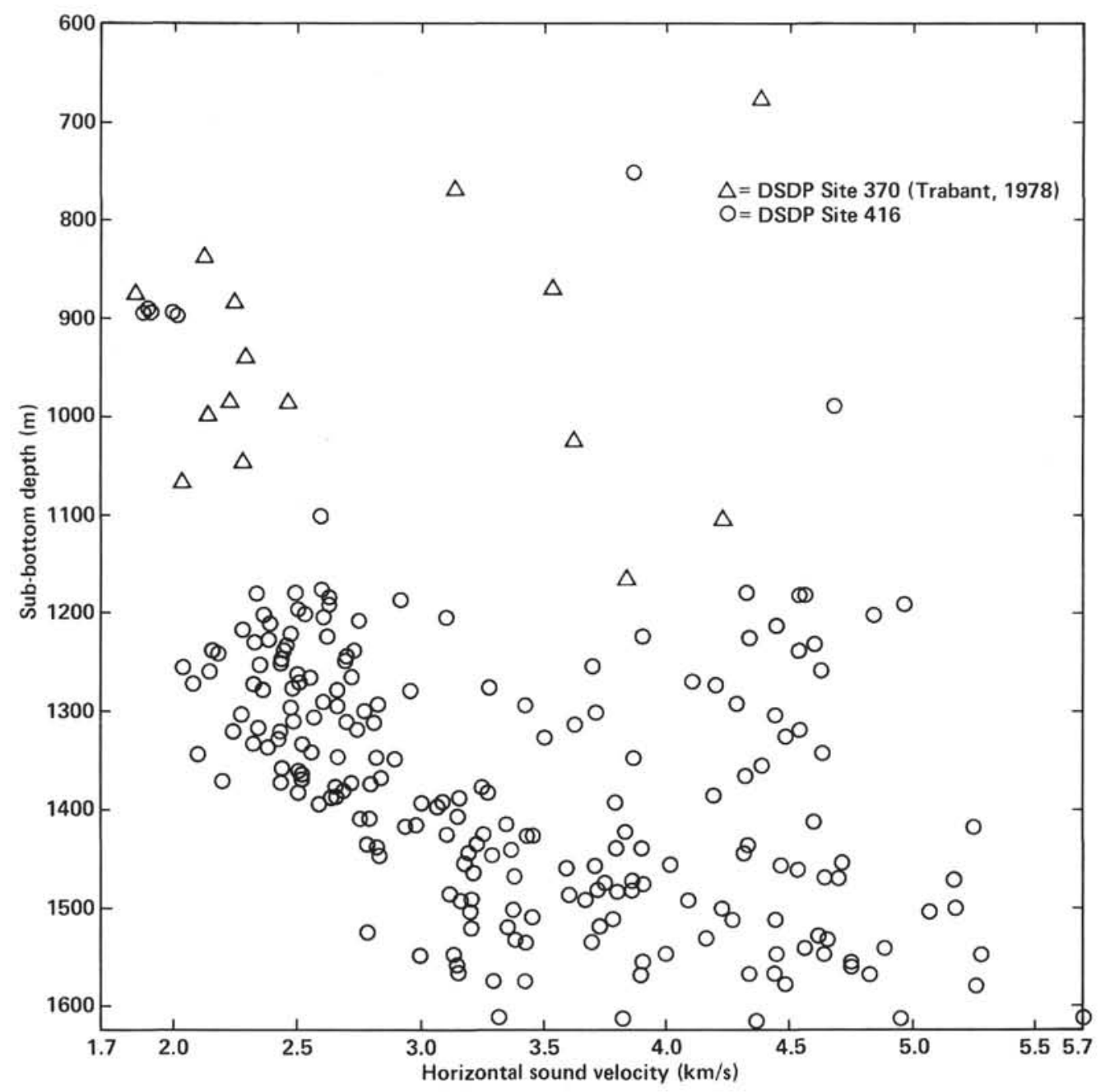

Figure 2. Horizontal velocity versus depth for the Cretaceous-Jurassic geologic section at Sites 416 and 370 . 


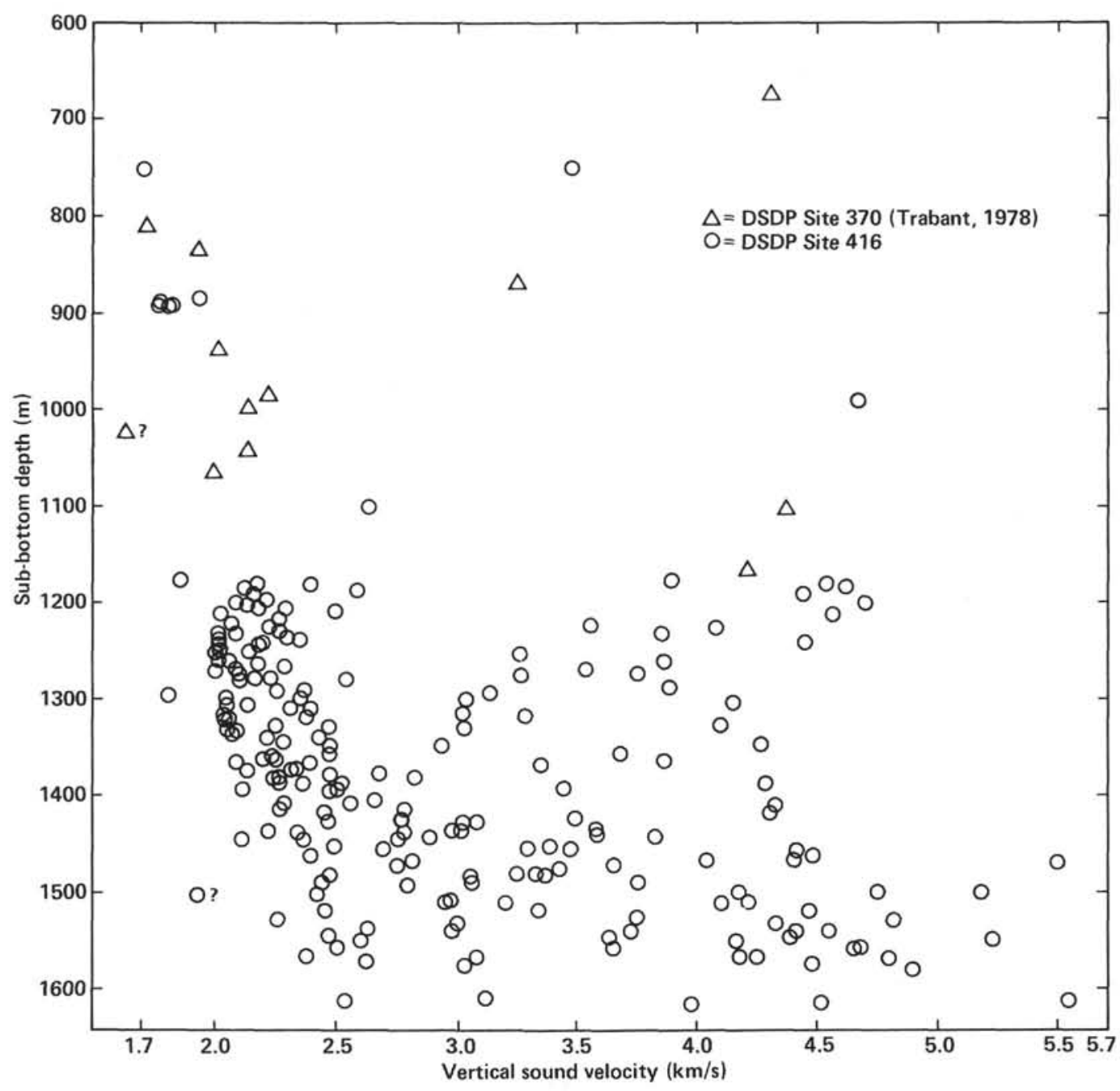

Figure 3. Vertical velocity versus depth for the Cretaceous-Jurassic geologic section at Sites 416 and 370 .

aligned with the bedding plane; $(4)$ foliation parallel to bedding, and (5) cementation along certain horizontal layers.

Figure 5 shows acoustic anisotropy for Cretaceous to Tithonian sandstone and siltstone turbidites in mudstone and minor limestone from 661 to $1624 \mathrm{~m}$ below the seafloor. In general, acoustic anisotropy of the sedimentary rocks below $1178 \mathrm{~m}$, which have velocities between about 2.0 and $4.2 \mathrm{~km} / \mathrm{s}$, is about $0.4 \mathrm{~km} / \mathrm{s}$ or more, faster in the horizontal than in the vertical plane. Some samples have an absolute anisotropy as great as 1.0 $\mathrm{km} / \mathrm{s}$. The relative acoustic anisotropy ranges from 0 to $30 \%, 5$ to $20 \%$ being typical. The mudstones which have velocities of 2.0 to $3.0 \mathrm{~km} / \mathrm{s}$, tend to have the greatest relative anisotropy, as compared with the higher velocity ( 3 to $4.2 \mathrm{~km} / \mathrm{s}$ ) sandstones, siltstones, and limestones. Where the sandstone, siltstone, and limestone have velocities greater than about $4.2 \mathrm{~km} / \mathrm{s}$, the acoustic anisotropy becomes much less significant, as the sample is more thoroughly cemented, and many samples are isotropic.

Figures 6 through 15 show the following physical properties versus depth for each lithologic type at Hole 416A: (1) vertical velocity and horizontal velocity (both at laboratory temperatures and pressures), (2) acoustic anisotropy, (3) porosity, (4) wet-bulk density, and (5) acoustic impedance. Mudstones are presented in Figures 6 and 7; siltstones, in Figures 8 and 9; sandstones, in Figures 10 and 11; marlstones, in Figures 12 and 13; and limestones, in Figures 14 and 15. Some of the calcarenites in Figures 10 and 11 are equivalent to limestone. Only the Hole 416A data listed in Table 1 are plotted, as the techniques used at Site $\mathbf{3 7 0}$ are not precisely comparable to the Site 416 data.

In Figure 7 the wet-bulk density and porosity of the mudstones are greater and less, respectively, than similar curves summarized in Hamilton (1976) for oceanic terrigenous sedimentary sequences. The fact that Site 416 data are different from Hamilton's could be in part related to differences in: (1) methods; (2) differences in grain-size distribution and mineralogy; (3) differences in the amount of calcium carbonate cement; (4) age of the Site 416 mudstones (being older, they have had more time to recrystallize, lithify, and to consolidate to a greater degree); or (5) overconsolidation of Site 416 mudstones by a theoretical, previously overlying sedimentary sequence which has been eroded away (Hedberg, 1936; Hamilton and Menard, 1956; Hamilton, 1959, 


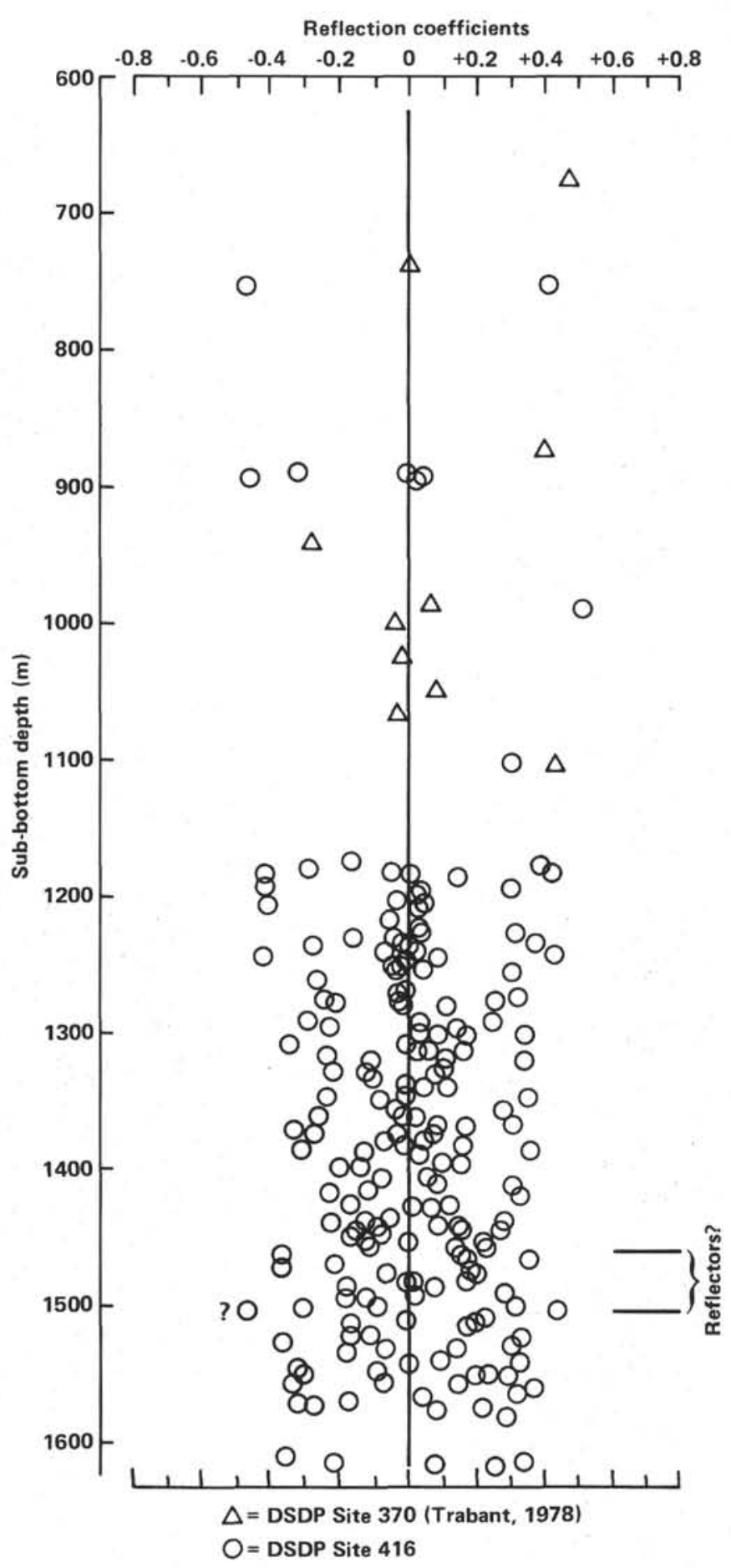

Figure 4. Reflection coefficient versus depth for the Cretaceous-Jurassic geologic section at Sites 416 and 370 .

1976, 1980; Margara, 1978). Of these possible causes, 1 through 4 are the most probable.

In Figure 7 there is a definite boundary at $1330 \mathrm{~m}$ where calcareous mudstones have a distinctly lower porosity than the overlying unit. Below $1450 \mathrm{~m}$ in Figure 6, mudstone acoustic anisotropy is at its greatest, which is what one would expect for mudstone as the clayey minerals become aligned horizontally and samples become more fissile (Press, 1966; Bachman, 1978).
Siltstones in Figures 8 and 9 show a distinct boundary at about $1425 \mathrm{~m}$. The siltstones are relatively uncemented above $1425 \mathrm{~m}$, and abundant calcareous cemented siltstones occur below $1425 \mathrm{~m}$, which have greater velocities and greater acoustic anisotropy. Irregular density-porosity variations are probably created by different grainsize distribution, grain packing, and different degrees of cementation.

Sandstone plots in Figures 10 and 11 show an uncemented sandstone trend and a cemented sandstone trend around $1200 \mathrm{~m}$ depth, with semilithified sandstones having a relatively high acoustic anisotropy. From 1200 $\mathrm{m}$ down the uncemented sandstone trend becomes more cemented and merges with the cemented sandstone trend (from 1200 to $1600 \mathrm{~m}$ ). Below 1300 or $1400 \mathrm{~m}$ the maximum relative acoustic anisotropy decreases down to $1550 \mathrm{~m}$. Below $1550 \mathrm{~m}$, porosity is typically around $5 \%$, sound velocity is very high, and acoustic anisotropy is relatively small. This is a result of the sandstones becoming so cemented (particularly the calcarenites) that they become more isotropic than the semicemented sandstone above. At $1420 \mathrm{~m}$ is the first high-horizontal-velocity-calcarenite $(>5.0 \mathrm{~km} / \mathrm{s})$. Below $1420 \mathrm{~m}$ sandstones appear to be more cemented.

The calcarenites in Figures 10 and 11 are essentially limestones, and they have typically greater velocities than the micritic limestone at the same horizon or depth. This is because they are more recrystallized.

Marlstone velocities in Figure 12 uniformly increase with increasing depth, but with a possible boundary at about $1530 \mathrm{~m}$.

Limestone velocity increases and porosity decreases with increasing depths in Figures 14 and 15. Many of the more cemented and lower porosity limestones have smaller relative acoustic anisotropies. Porosities here range from 4 to $18 \%$, which are lower than porosities of Cretaceous Pacific Ocean pelagic nannofossil-foraminiferal limestones (Schlanger, Jackson et al., 1976), where identical laboratory methods were used. However, Site 416 limestones are micritic, older, and more deeply buried; thus they are more recrystallized and probably should have a smaller porosity than the nannofossil-foraminifer types. Site 416 limestone porosities are also slightly lower than the limestone porosity-depth plot summarized by Scholle (1977).

\section{In Situ Reflection Velocity}

In general, from 661 to $880 \mathrm{~m}$, the Albian to Barremian claystone, with some limestone, sandstone, and siltstone, have velocities ranging from 1.70 to $4.37 \mathrm{~km} / \mathrm{s}$. According to Boyce (1980b) an in situ average vertical velocity is estimated to be $1.93 \mathrm{~km} / \mathrm{s}$.

From 880 to $1430 \mathrm{~m}$, the Hauterivian to Valanginian turbidites of alternating graded calcareous and quartzose cycles from siltstone or fine sandstone to mudstone have velocities ranging from 1.80 to $4.96 \mathrm{~km} / \mathrm{s}$. Boyce (1980b) estimated an in situ average vertical velocity of $2.61 \mathrm{~km} / \mathrm{s}$.

From 1430 to $1624 \mathrm{~m}$, the early Valanginian to Tithonian (Kimmeridgian) turbidites with alternating quartzose siltstone grading to mudstone cycles with hard mi- 


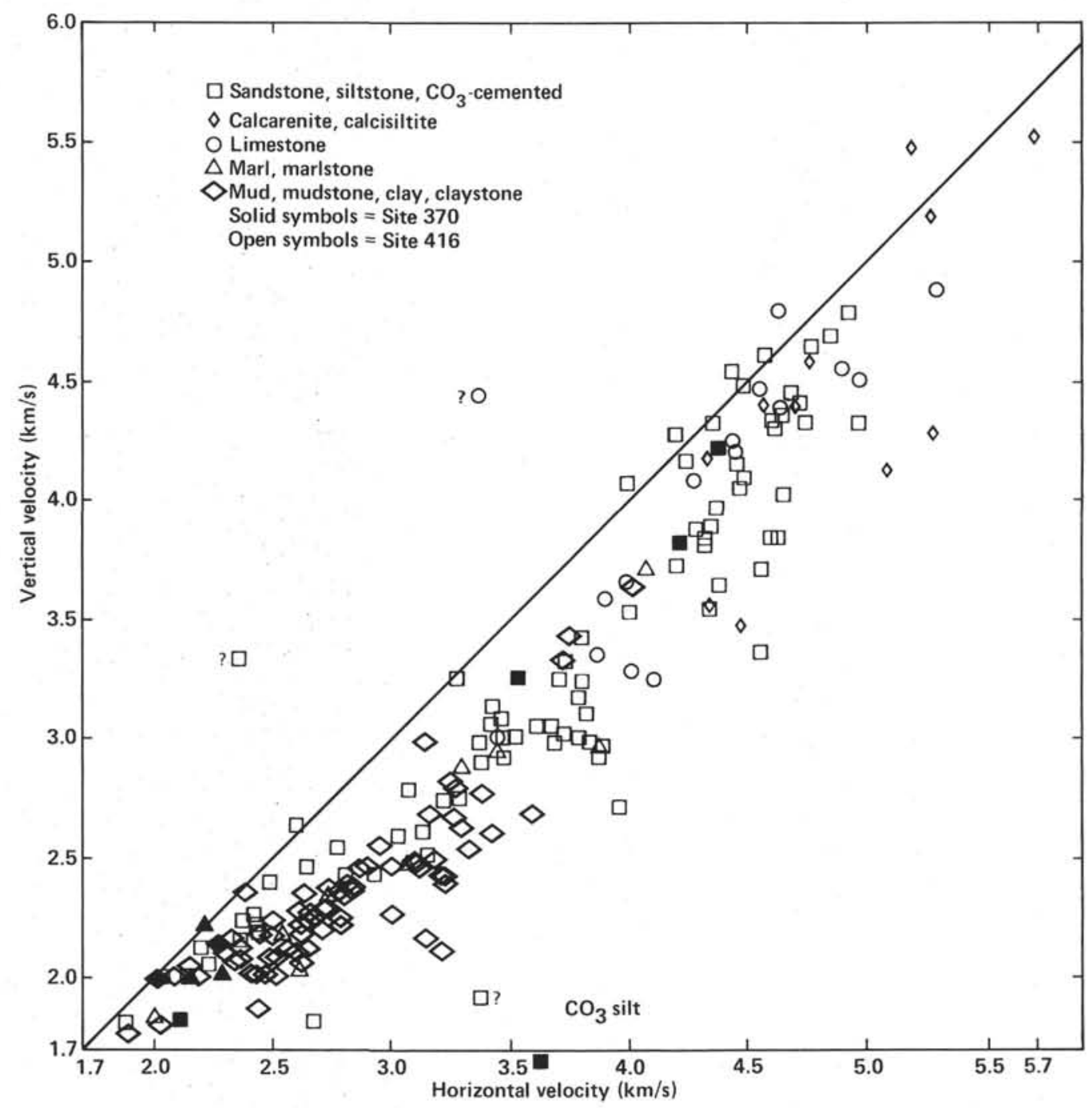

Figure 5. Vertical velocity versus horizontal velocity for the Cretaceous-Jurassic geologic section at Sites 416 and 370 .

critic limestone and calcarenite (calciturbidites) have velocities from 2.26 to $5.7 \mathrm{~km} / \mathrm{s}$. According to Boyce (1980b) the unit has an in situ average vertical velocity of $3.25 \mathrm{~km} / \mathrm{s}$ (see footnote 2). These in situ velocities are based on logging velocities and laboratory velocities. The results are very subjective, for: (1) the percentages of each rock type must be known, (2) he (Boyce) must fill in data where coring data are sparse, (3) log velocities tend to be biased too low, and (4) there is no caliper data to judge the variability of the sonic log.

If we assume that Boyce's (1980b, Table 6) estimated in situ velocities and reflection time (round-trip) from 0 to $1616 \mathrm{~m}$ of $1.50 \mathrm{~s}$ are correct, ${ }^{3}$ then the 1.43 -s-reflector discussed by Lancelot, Winterer et al. (1980b) would correlate at about $1500 \mathrm{~m}$ in the geologic section at Hole

\footnotetext{
${ }^{2}$ The text of Boyce (1980b), p. 316 , incorrectly lists " $3.75 \mathrm{~km} / \mathrm{s}$ " for the velocity of the Tithonian to Valanginian sandstone, siltstone, marlstone, and limestone from 1430 to $1624 \mathrm{~m}$; it should be $3.25 \mathrm{~km} / \mathrm{s}$ as in Table 6. This also applies to p. 150 in Lancelot, Winterer et al., $1980 \mathrm{~b}$.

${ }^{1980 \mathrm{~b}}$ Hole 516 penetrated to $1624 \mathrm{~m}$; however, because of less than $100 \%$ recovery and DSDP depth conventions, the deepest velocity measurement is at $1616 \mathrm{~m}$.
}

416A, where there is a major change in the acoustical character of the sequence at about a quarter wavelength above $1500 \mathrm{~m}$ (Fig. 3).

Lancelot, Winterer (1980b) believe this 1.43-s reflector is actually below $1624 \mathrm{~m}$ at Site 416 , based on stacking velocities and general geologic knowledge of the area. They may be correct, for the data here are also highly subjective. While these results do not disprove the conclusions reached by Lancelot, Winterer et al. (1980b), they do offer another possible interpretation to add to those already discussed in Lancelot, Winterer et al. (1980b) and Lancelot and Winterer (1980) and point to the difficulty of correlating seismic reflection data to drill hole data. Lancelot and Winterer (1980) interpreted the 1.43-s reflector (blue reflector) to represent a Callovian-Oxfordian transgressive phase of more pelagic sediment character. If the blue reflector actually correlates to $1500 \mathrm{~m}$ in Hole $416 \mathrm{~A}$, then the Callovian-Oxfordian transgressive sedimentary sequence is not represented by this blue reflector, but may occur as a "deeper" reflector in the seismic section. 


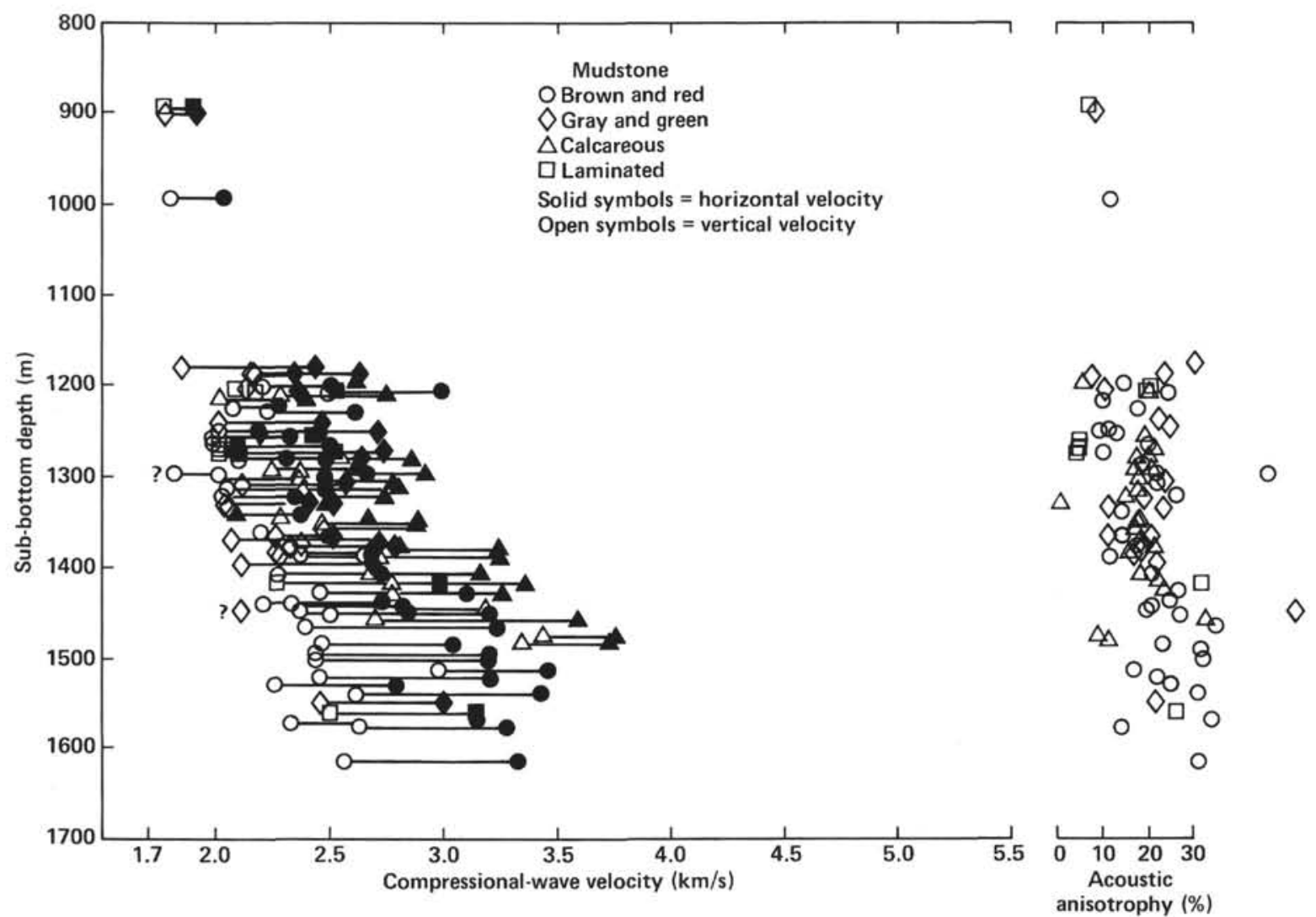

Figure 6. Mudstone velocities and acoustic anisotropy versus depth for the Cretaceous-Jurassic geologic section at Site 416.

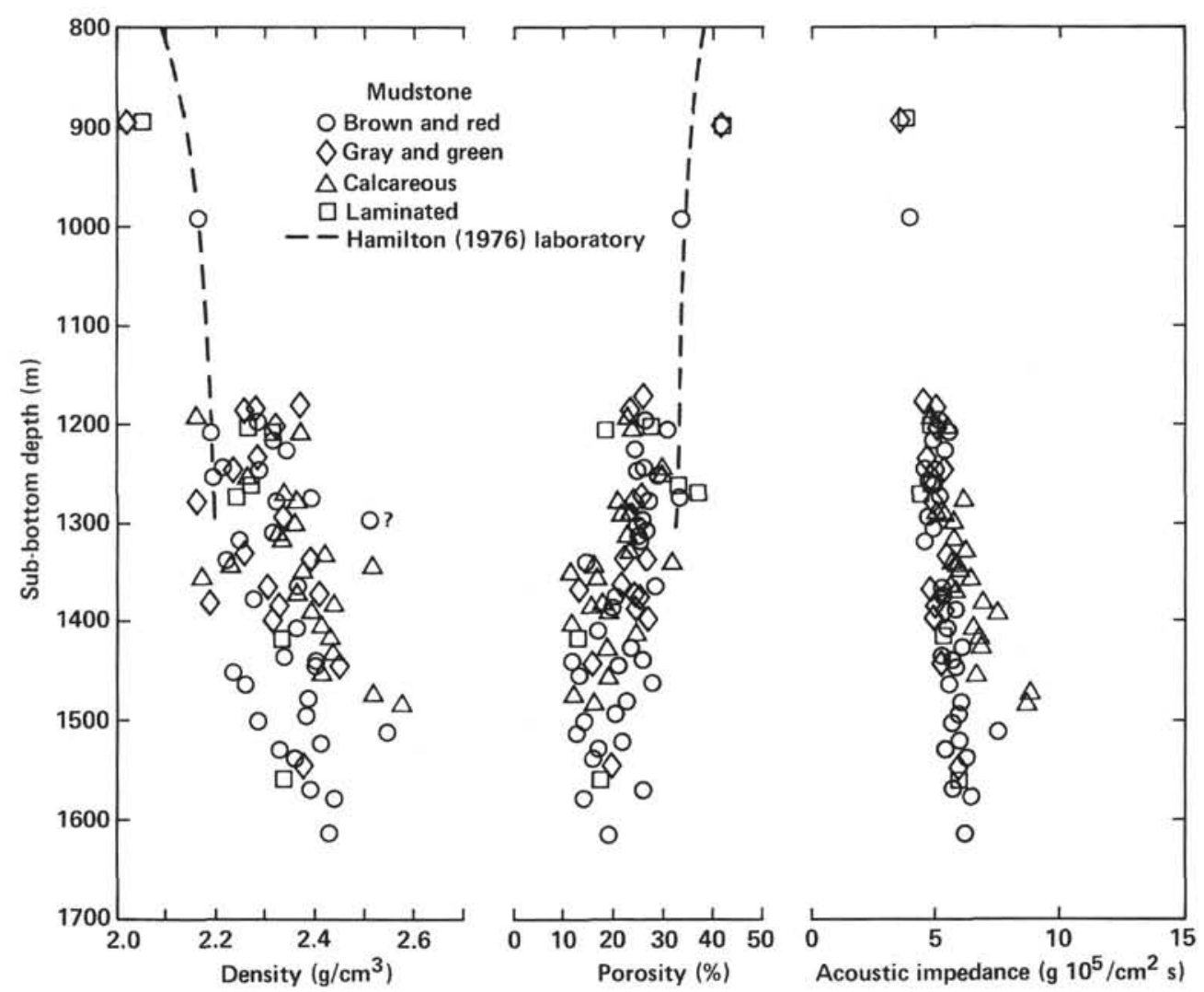

Figure 7. Mudstone density, porosity, and acoustic impedance versus depth for the Cretaceous-Jurassic geologic section at Site 416 . 


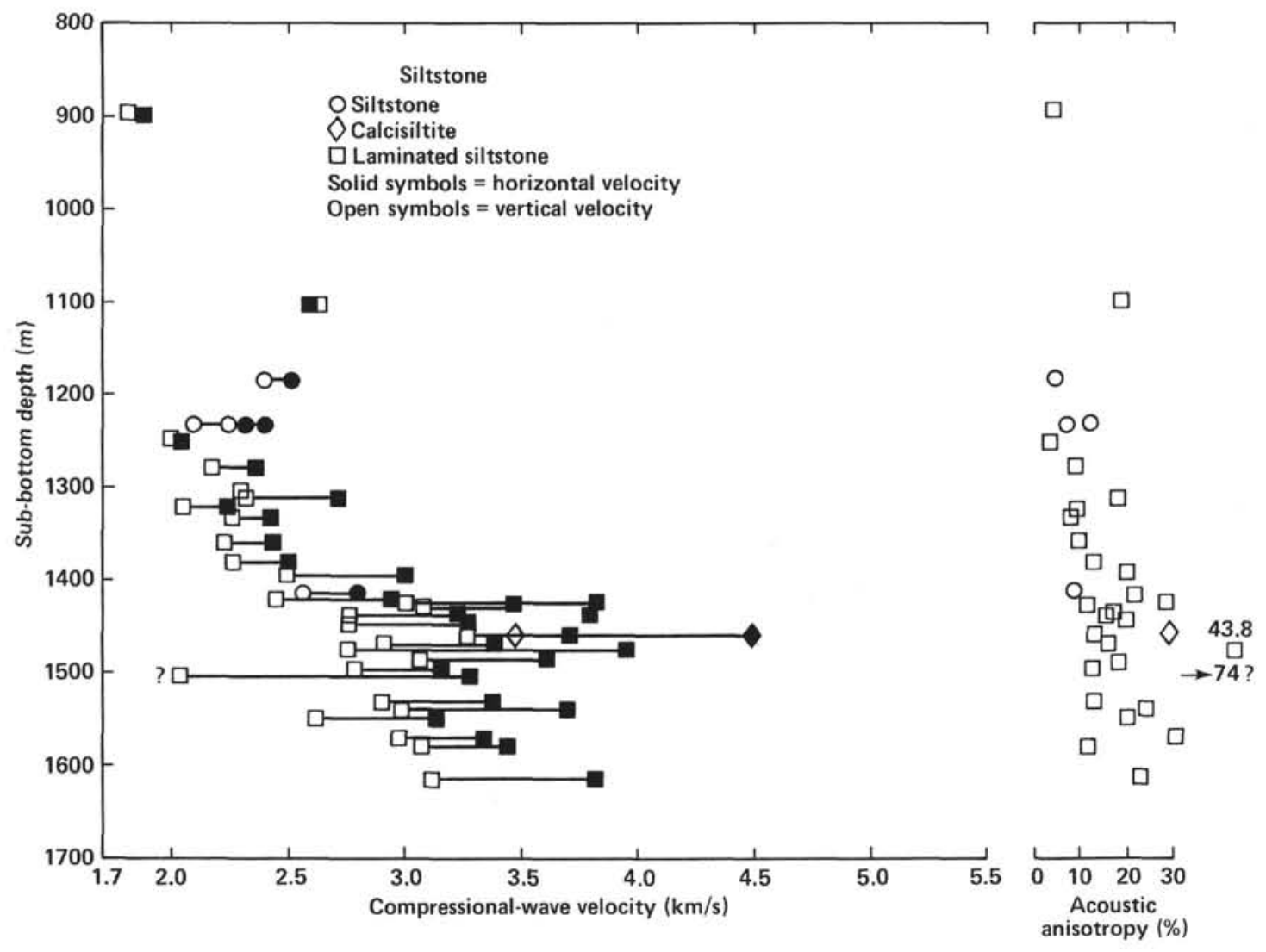

Figure 8. Siltstone velocities and acoustic anisotropy versus depth for the Cretaceous-Jurassic geologic section at Site 416.

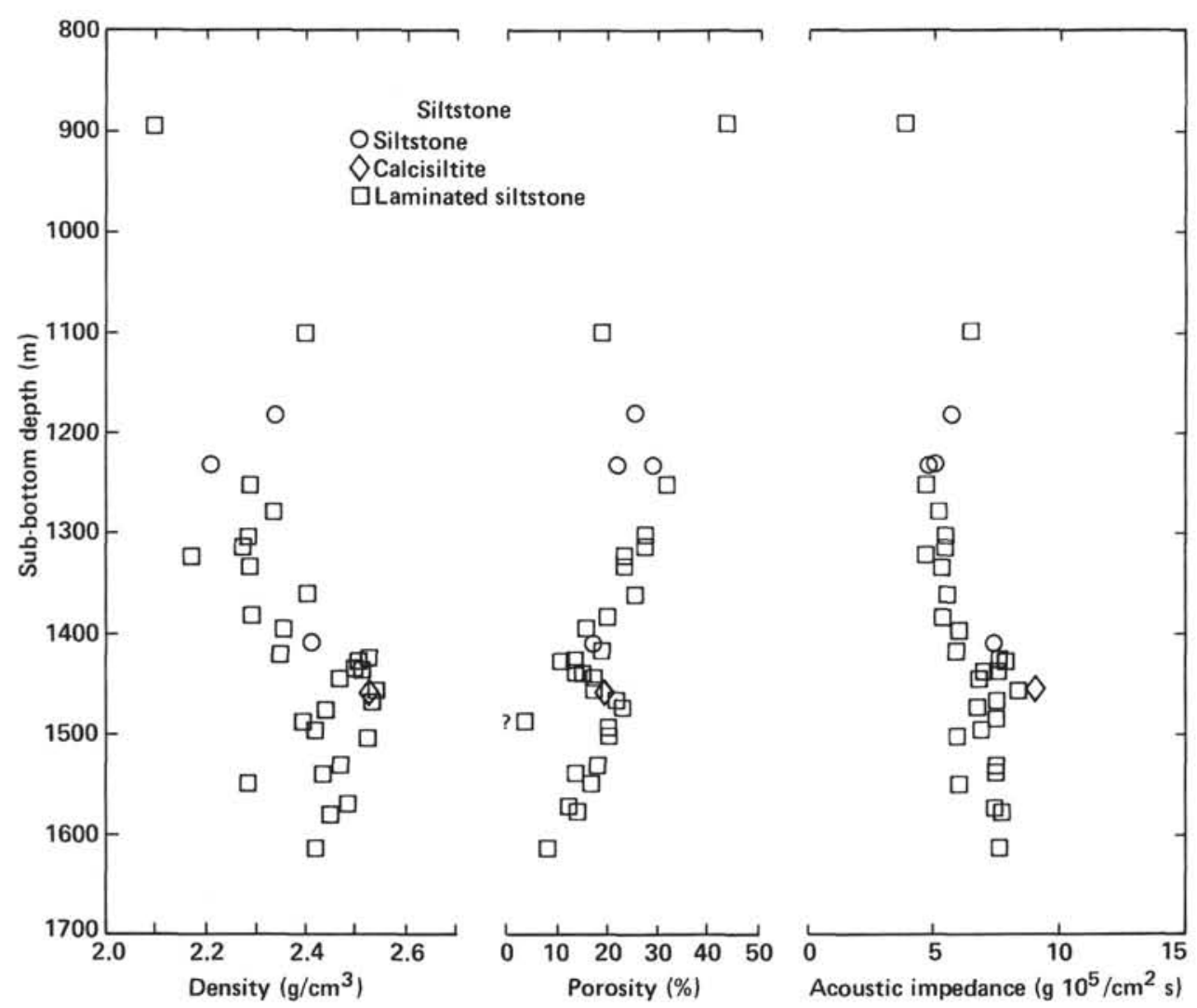

Figure 9. Siltstone density, porosity, and acoustic impedance versus depth for the Cretaceous-Jurassic geologic section at Site 416 . 
R.E. BOYCE

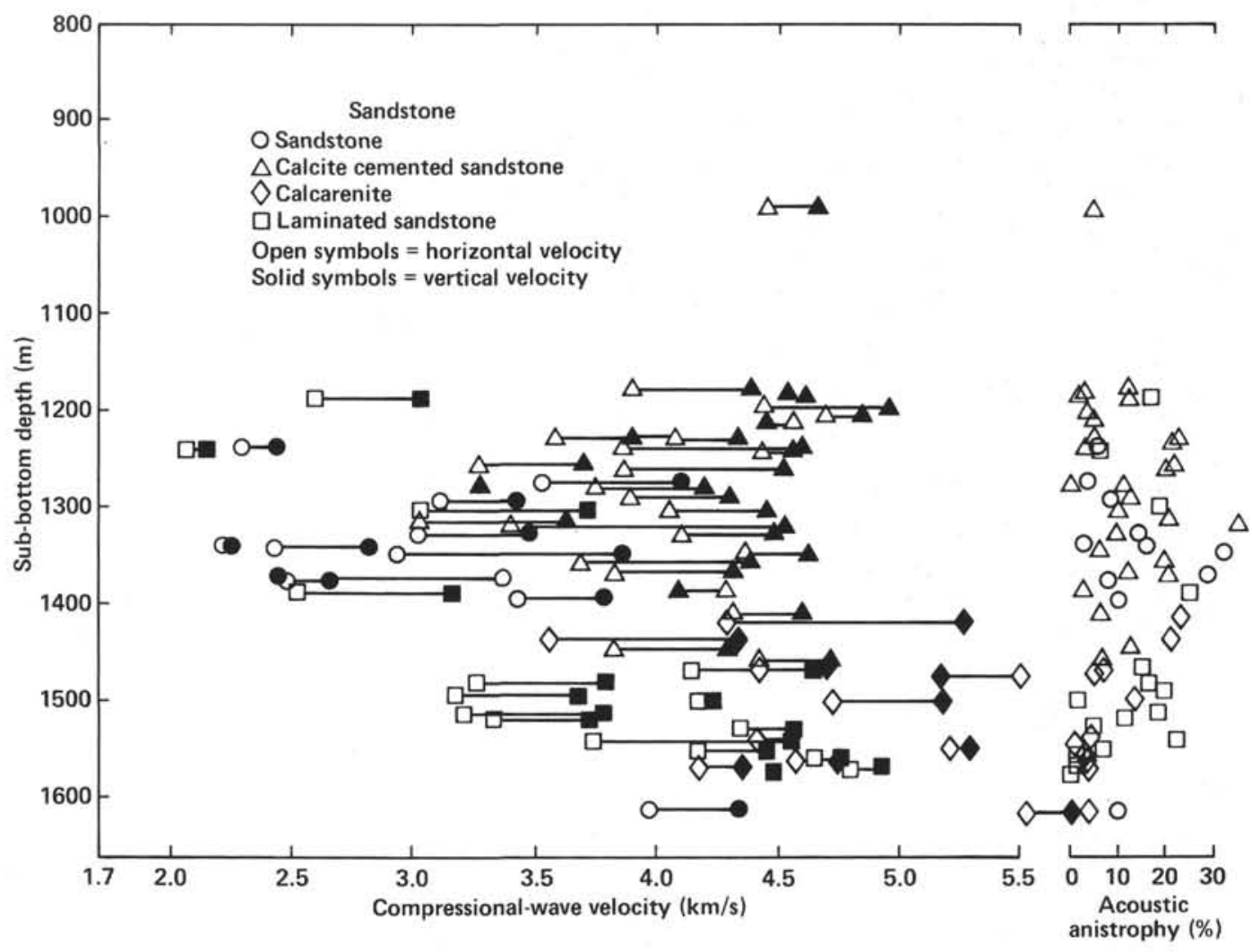

Figure 10. Sandstone velocities and acoustic anisotropy versus depth for the Cretaceous-Jurassic geologic section at Site 416.

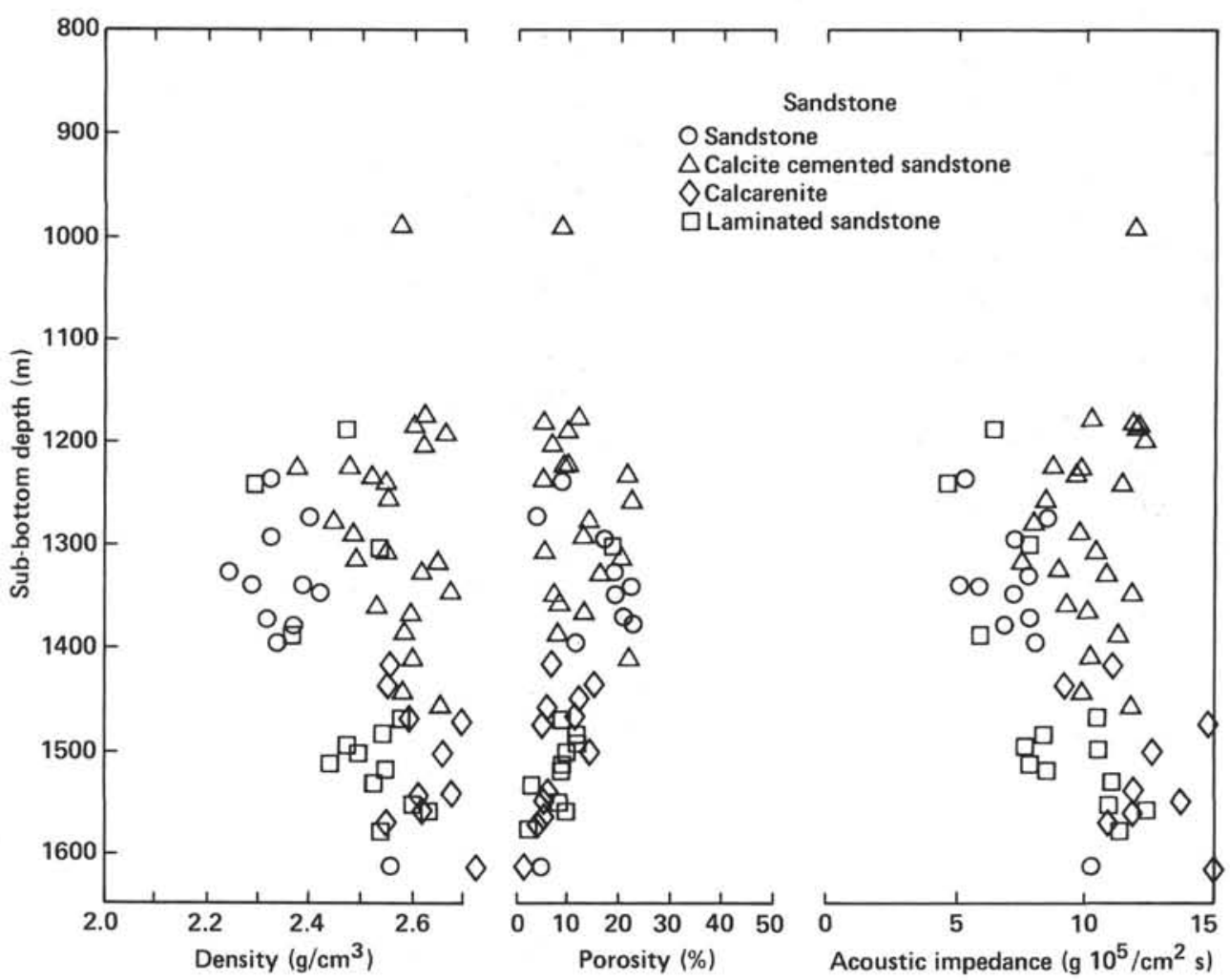

Figure 11. Sandstone density, porosity, and acoustic impedance versus depth for the Cretaceous-Jurassic geologic section at Site 416 . 


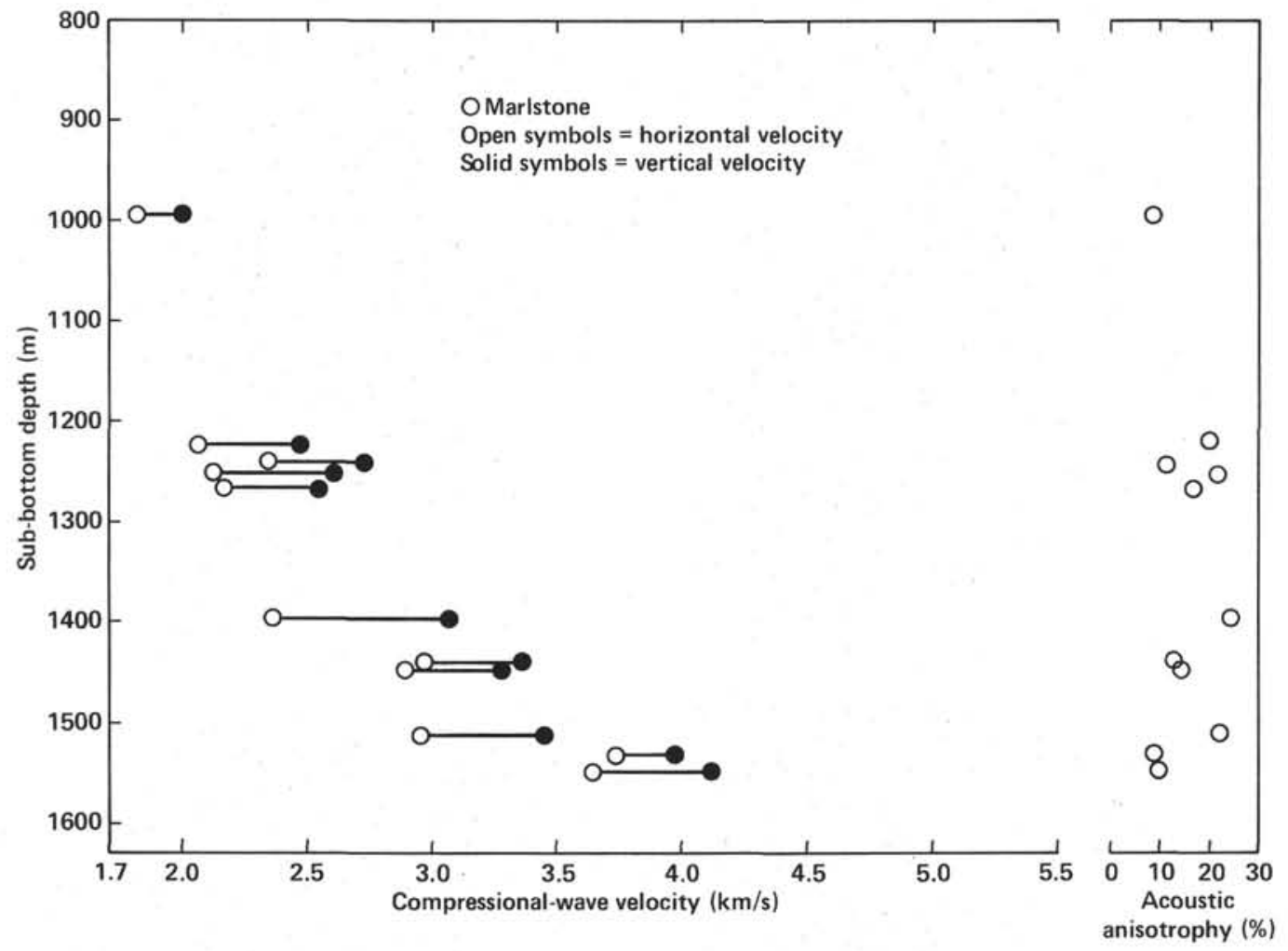

Figure 12. Marlstone velocities and acoustic anisotropy versus depth for the Cretaceous-Jurassic geologic section at Site 416 .

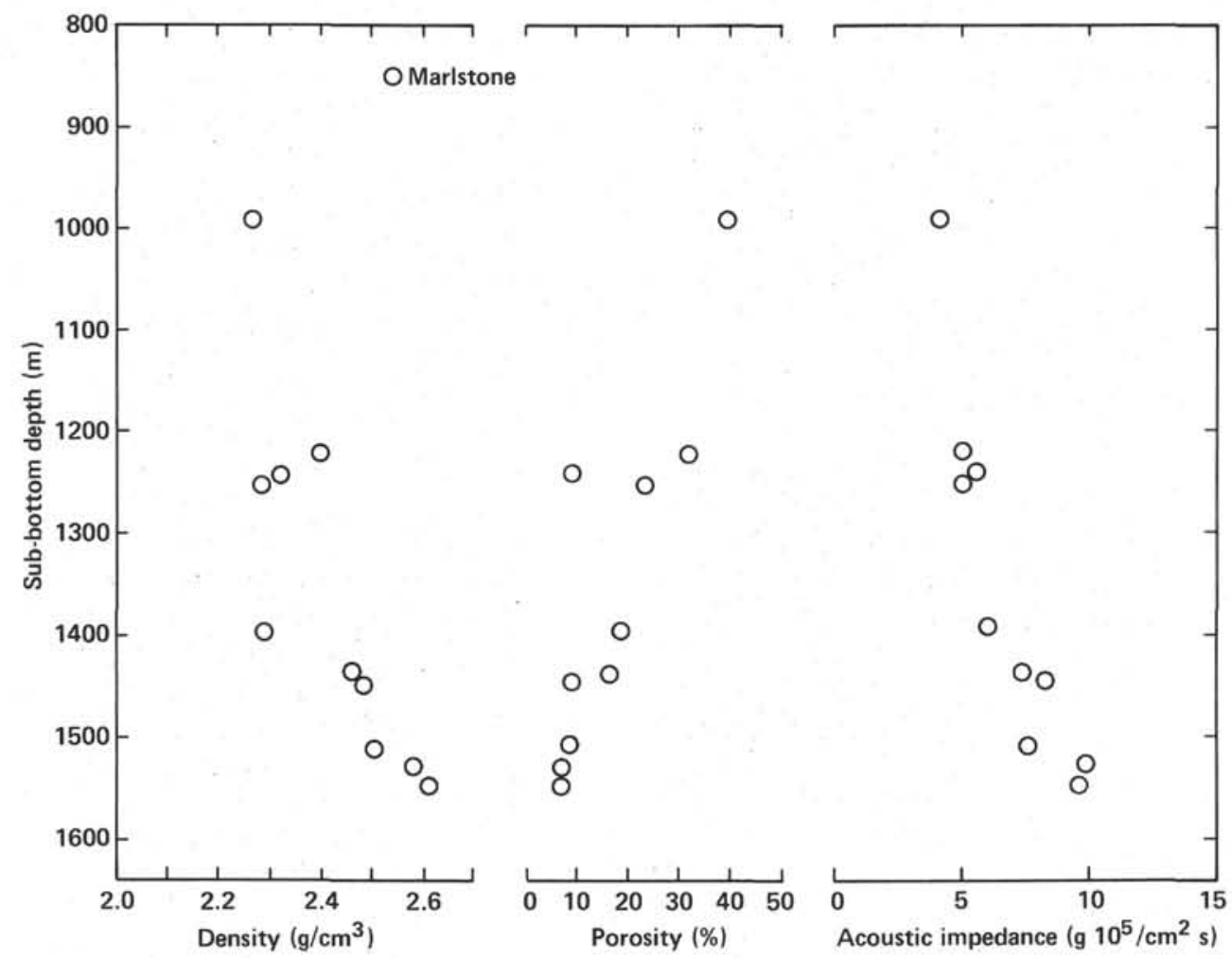

Figure 13. Marlstone density, porosity, and acoustic impedance versus depth for the Cretaceous-Jurassic geologic section at Site 416 . 

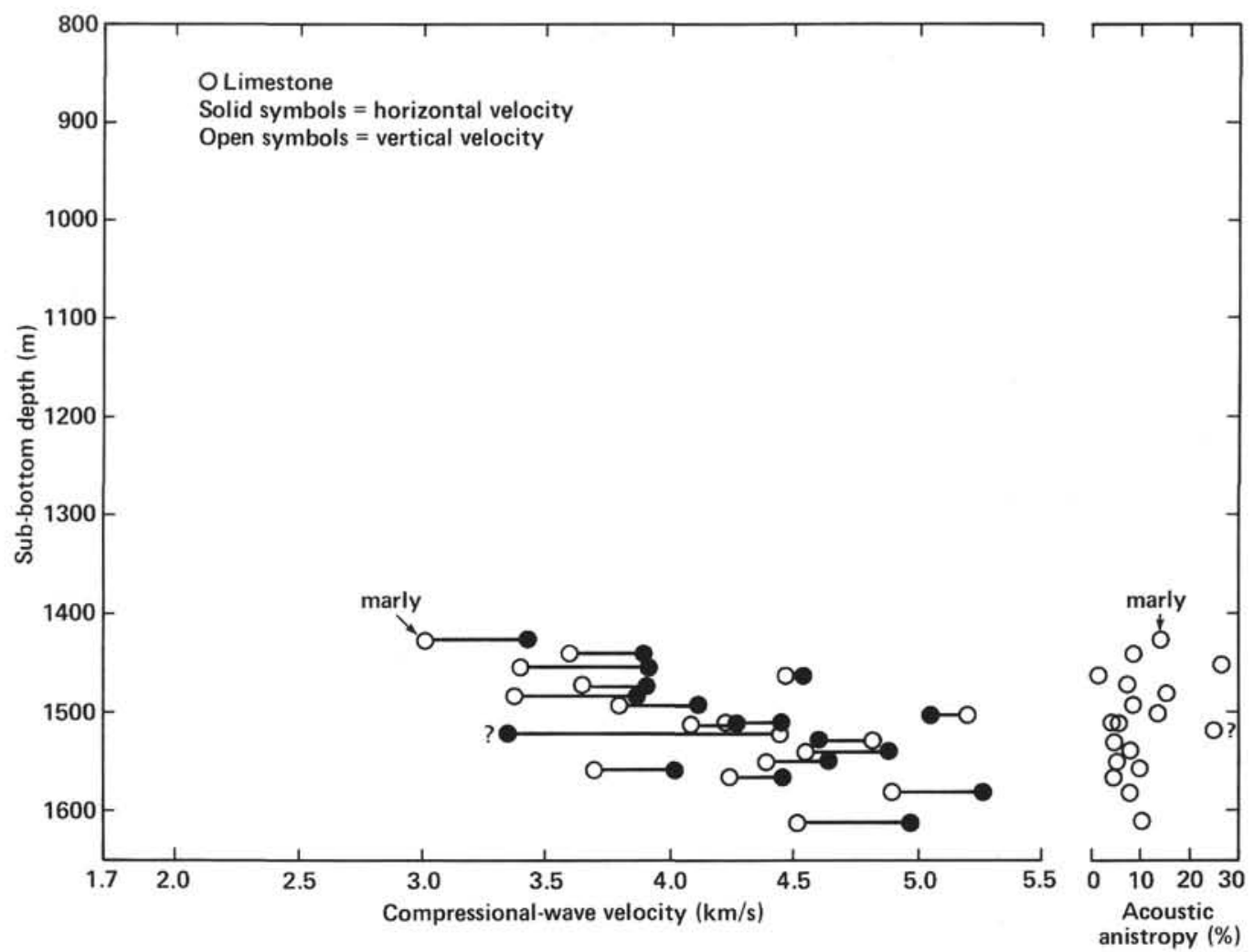

Figure 14. Limestone velocities and acoustic anisotropy versus depth for the Cretaceous-Jurassic geologic section at Site 416.
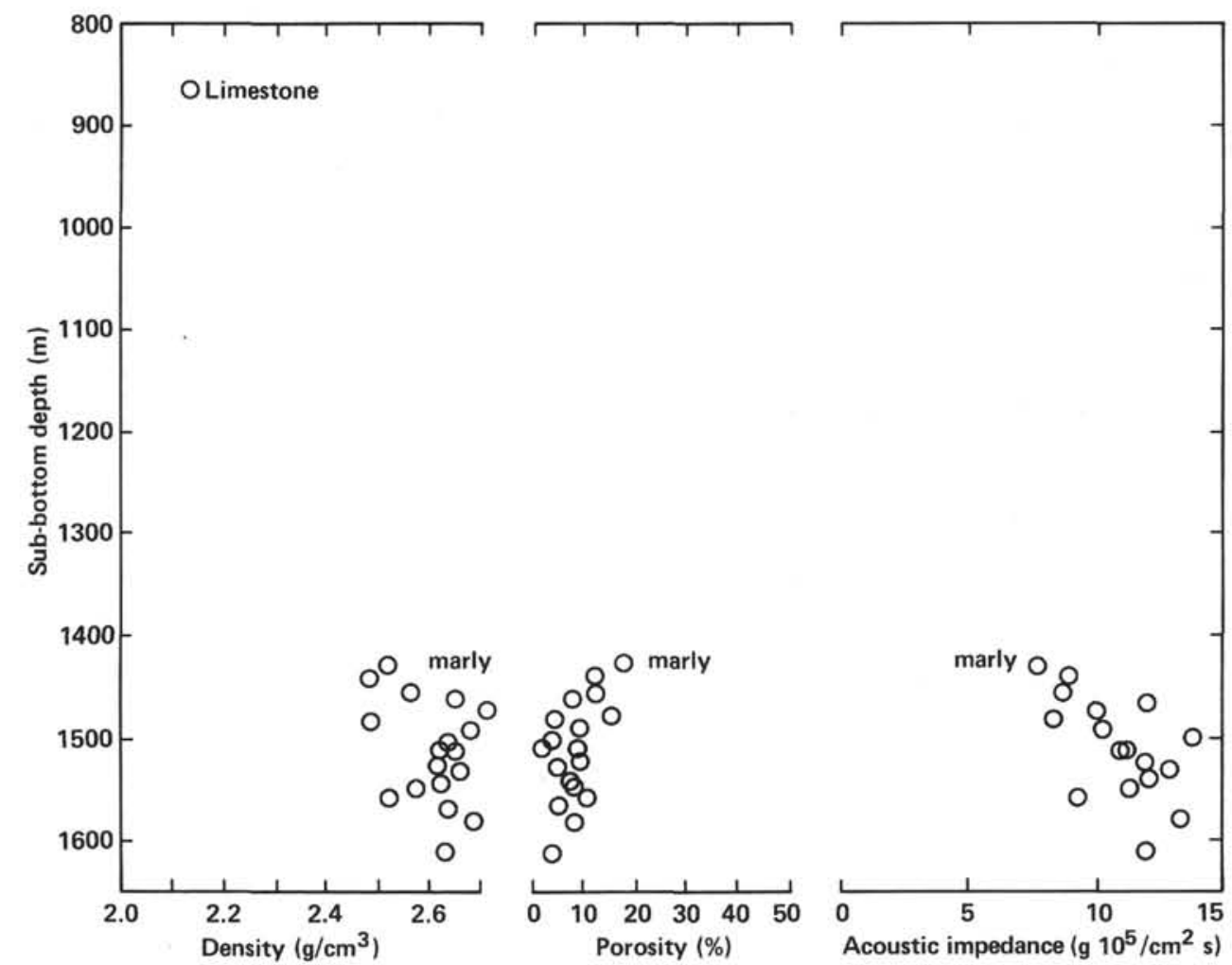

Figure 15. Limestone densities, porosity, and acoustic impedance versus depth for the CretaceousJurassic geologic section at Site 416. 


\section{Comparison to Site 530 Cretaceous Rocks}

The oldest sediment recovered at Site 530 is Cenomanian. These are younger than any of the Cretaceous-Jurassic (Albian to Tithonian) sediments recovered at Sites 370 and 416 . In addition, data from Sites 370 and 416 extend to a much greater depth below the seafloor (1624 $\mathrm{m}$ vs. $1103 \mathrm{~m}$ ). Therefore, one would expect these to be more lithified than sediments at Site 530 .

The greater degree of lithification at Sites 370 and 416 compared to Site 530 is suggested by comparing mudstone-porosity versus depth curves from each area (Boyce, this volume). At Site 530, Hamilton's (1976) summary curve matches the data very well, while at Sites 370 and 416 Hamilton's (1976) mudstone-porosity curve versus depth does not match. Mudstone porosities at Sites 370 and 416 are less than Hamilton's curve, suggesting either (1) different methods; (2) different mineralogy, grain-size, packing and cementation; (3) or overconsolidation at Sites 370 and 416 by a geologic section which has now been eroded away. This does not appear to be the case at Site 530, which appears to be normally consolidated, and mudstone lithologies and grain size may be more similar to those mudstones discussed in Hamilton (1976). Velocities of mudstone are generally higher at Sites 416 and 370.

Limestone porosities at Site 530 are about $15 \%$, which compares well with limestones at Sites 370 and 416. In addition, at Site 530, limestone velocities are within the limestone-velocity range at Sites 416 and 370.

Porosities of carbonate-cemented siliclastic sandstones compare well at both Site 530 and Sites 370/416. Porosities are $\sim 10$ to $\sim 12 \%$. Velocities of carbonatecemented sandstone of both areas are also similar.

The acoustic anisotropy of the two areas is, however, quite different. The anisotropy at Site $\mathbf{5 3 0}$ is relatively low compared with all of the data from Sites 370 and 416. At Site 530 , anisotropy is typically about $0.2 \mathrm{~km} / \mathrm{s}$, which is generally similar to anisotropy at other DSDP sites (Bachman, 1978), and it even compares well to Sites 370 and 416 above $1178 \mathrm{~m}$. However, below 1178 $\mathrm{m}$ at Sites 416 and 370, anisotropy is very large (about $0.4 \mathrm{~km} / \mathrm{s}$ or more) where velocities are between 2.2 and $4.2 \mathrm{~km} / \mathrm{s}$. In part this could be related to a more calcareous cementation or to greater lithification at Sites $\mathbf{3 7 0}$ and 416. However, the calcareous-cement concept also explains the calcareous mudstones at Site 530, which had significantly higher $(37 \%)$ anisotropies than uncemented mudstone.

\section{SUMMARY AND CONCLUSIONS}

From 661 to $880 \mathrm{~m}$ are Albian to Barremian claystone with some limestone, sandstone, and siltstone. Compressional-wave velocities ranged from 1.70 to $4.37 \mathrm{~km} / \mathrm{s}$, with an average in situ vertical velocity of $1.93 \mathrm{~km} / \mathrm{s}$.

From 880 to $1430 \mathrm{~m}$ are Hauterivian to Valanginian turbidites of alternating graded calcareous and quartzose cycles from siltstone or fine sandstone to mudstone. Compressional-wave velocities range from 1.80 to 4.96 $\mathrm{km} / \mathrm{s}$, with an average in situ velocity of $2.61 \mathrm{~km} / \mathrm{s}$.

From 1430 to $1624 \mathrm{~m}$ are early Valanginian to Tithonian (Kimmeridgian?) turbidites with alternating quartz- ose siltstone grading to mudstone cycles with hard micrite and calcarenite (calciturbidites). Compressionalwave velocities range from 2.26 to $5.7 \mathrm{~km} / \mathrm{s}$, with an average in situ vertical velocity of $3.25 \mathrm{~km} / \mathrm{s}$

Acoustic anisotropy is 0 to $30 \%$ in the Cretaceous to Tithonian sandstone-siltstone turbidites in mudstone and minor limestone from 661 to $1624 \mathrm{~m}$ below the seafloor. Between 2.0(?) km/s and 4.2(?) km/s, anisotropy becomes particularly significant (below $1178 \mathrm{~m}$ ), where the anisotropy is about $+0.4 \mathrm{~km} / \mathrm{s}$ or greater. The mudstone, softer sandstone, and softer siltstone tend to have velocities around 2.0 to $2.5 \mathrm{~km} / \mathrm{s}$; the cemented sandstone and limestone cluster about $2.5 \mathrm{~km} / \mathrm{s}$ to 4.2 $\mathrm{km} / \mathrm{s}$; thus the relative percentage anisotropy is greater for the lower-velocity lithologies. Above $4.2(?) \mathrm{km} / \mathrm{s}$, the well-cemented sandstone and limestone tend to have a smaller (less than $0.4 \mathrm{~km} / \mathrm{s}$ ) absolute anisotropy, and many samples are nearly isotropic. The anisotropy can be related to some combination of the following: (1) elongated or platy grains, which provide a faster path horizontally as a result of there being fewer gaps between minerals, (2) preferred orientation of minerals which have an acoustic anisotropy, (3) cementation along certain horizontal layers, (4) alternating high- and low-velocity layers, and (5) a larger number of horizontal cracks or foliation.

The mudstone's porosity and wet-bulk density curves versus depth are slightly higher and lower, respectively, for similar porosity and density curves in Hamilton (1976). This could be the result of some combination of methods, age, and lithology differences, or overconsolidation by an overlying geologic section which has been eroded away.

Limestone porosities are typically less than Cretaceous limestones from the Pacific Ocean (Schlanger, Jackson et al., 1976), where identical methods were used. These Pacific limestones are the nannofossil foraminifer type, and the limestone from Site 416 is micritic; thus the micritic limestone is probably more recrystallized and thus could perhaps be expected to have a smaller porosity.

If the average in situ vertical velocities calculated by Boyce (1980b) for Hole 416A are correct, then the 1.43-s (round-trip) reflector (blue) discussed by Lancelot and Winterer (1980) and Lancelot, Winterer et al. (1980b) would correlate to about $1500 \mathrm{~m}$ in Hole 416A, and not below $1624 \mathrm{~m}$ as discussed by Lancelot and Winterer (1980c). There does appear to be a significant change in the acoustic character at or around $1500 \mathrm{~m}$ (early Valanginian) to a more calcareous and cemented lithology. This investigator only suggests another possible interpretation, since this one is based on many assumptions and is highly subjective.

\section{ACKNOWLEDGMENTS}

The author wishes to thank Dr. E. L. Hamilton and Dr. Thomas H. Shipley for reviewing the manuscript.

\section{REFERENCES}

Bachman, R. T., 1979. Acoustic anisotropy in marine sediments and sedimentary rocks. J. Geophys. Res., 84(1113):7467-7663.

Birch, F., 1961. The velocity of compressional waves in rocks to 10 kilobars, Part 2. Geophys. Res., 66:2199.

Boyce, R. E., 1980a. Deep Sea Drilling Project Leg 50 laboratory physical-property methods. In Lancelot, Y., Winterer, E. L., et al., In- 


\section{R. E. BOYCE}

it. Repts. DSDP, 50: Washington (U.S. Govt. Printing Office), 837-848.

1980b. Determination of the relationships of electrical resistivity, sound velocity, and density/porosity of sediment and rock by laboratory techniques and well logs from Deep Sea Drilling Project Sites 415 and 416 off the coast of Morocco. In Lancelot, Y., Winterer, E. L., et al., Init. Repts. DSDP, 50: Washington (U.S. Govt. Printing Office), 305-318.

Bullen, K. E., 1947. Introduction to the Theory of Seismology: London (Cambridge University Press).

Carlson, R. L., and Christensen, N. I., 1977. Velocity, anisotropy, and physical properties of deep-sea sediments from the western South Atlantic. In Supko, P. R., Perch-Nielsen, K., et al., Init. Repts. DSDP, 39: Washington (U.S. Govt. Printing Office), 555-559.

Evans, H. B., 1965. GRAPE-A device for continuous determination of material density and porosity. SPWIA Logging Symp., Sixth Ann. Trans. (Dallas, Texas), 2:B1-B25.

Gregory, A. R., 1977. Aspects of rock physics from laboratory and $\log$ data that are important to seismic interpretation. In Payton, C. H. (Ed.), Seismic Stratigraphy-Applications to Hydrocarbon Exploration, AAPG Mem., 26:15-46.

Hamilton, E. L., 1959. Thickness and consolidation of deep sea sediments. Geol. Soc. Am. Bull., 70:1399-1424.

1971a. Elastic properties of marine sediments. J. Geophys. Res., 76:579-604.

1971b. Prediction of in situ acoustic and elastic properties of marine sediments. Geophysics, 36:266-284.

1976. Variations of density and porosity with depth in deepsea sediments. J. Sediment Petrol., 46(2):280-300.

1978. Sound velocity-density relations in sea floor sediments and rocks. J. Acoust. Soc. Am., 63(2):366-377.

1980. Geoacoustic modeling of the sea floor. J. Acoust. Soc. Am., 68:1313-1340.

Hamilton, E. L., and Menard, H. W., 1956. Density and porosity of sea floor surface sediments off San Diego, California. AAPG Bull., 40(4):754-761.

Hedberg, H. D., 1936. The gravitational compaction of clays and shales. Am. J. Sci., 231:241-287.

Lancelot, Y., Seibold, E., and Gardner, J. V., 1978. Introduction. In Lancelot, Y., Seibold, E., et al., Init. Repts. DSDP, 41: Washington (U.S. Govt. Printing Office), 7-18.
Lancelot, Y., Seibold, E., et al., 1978. Site 370: Deep basin off Morocco. In Lancelot, Y., Seibold, E., et al., Init. Repts. DSDP, 41: Washington (U.S. Govt. Printing Office), 421-492.

Lancelot, Y., and Winterer, E. L., 1980. Evolution of the Moroccan Ocean Basin and adjacent continental margin-A synthesis. In Lancelot, Y., Winterer, E. L., et al., Init. Repts. DSDP, 50: Washington (U.S. Govt. Printing Office), 801-822.

Lancelot, Y., Winterer, E. L., et al., 1980a. Explanatory notes and shipboard procedures, Deep Sea Drilling Project Leg 50. In Lancelot, Y., Winterer, E. L., et al., Init. Repts. DSDP, 50: Washington (U.S. Govt. Printing Office), 13-28.

1980b. Site 416, in the Moroccan Basin, Deep Sea Drilling

Project Leg 50. In Lancelot, Y., Winterer, E. L., et al., Init. Repts. DSDP, 50: Washington (U.S. Govt. Printing Office), 115-302.

Laughton, A. S., 1957. Sound propagation in compacted ocean sediments. Geophysics, 22:233-260.

Magara, K., 1978. Compaction and Fluids Migration Practical Petroleum Geology: New York (Elsevier).

Nafe, J. E., 1963. Physical properties of marine sediments. In Hill, M. N. (Ed.), The Sea: (Vol. 3): New York (Interscience), p. 749.

Nafe, J. E., and Drake, C. L., 1957. Variation with depth in shallow and deep water marine sediments of porosity density and the velocities of compressional and shear waves. Geophysics, 22:523-552.

Press, F., 1966. Seismic velocities. In Clark, S. P. (Ed.), Handbook of Physical Constants, Mem. Geol. Soc. Am., 97:195.

Schlanger, S. O., Jackson, E. D., et al., 1976. Site 316. In Schlanger, S. O., Jackson, E. D., et al., Init. Repts. DSDP, 33: Washington (U.S. Govt. Printing Office), 105-160.

Scholle, P. A., 1977. Chalk diagenesis and its relation to petroleum exploration: Oil from chalks, a modern miracle? AAPG Bull., 61: 982-1009.

Trabant, P. K., 1978. Synthesis of physical properties data from DSDP Leg 41. In Lancelot, Y., Seibold, E. et al., Init. Repts. DSDP, 41: Washington (U.S. Govt. Printing Office), 1199-1214.

Wood, A. B., 1941. A Textbook of Sound: New York (MacMillan).

Wyllie, M. R. J., Gregory, H. R., and Gardner, L. W., 1956. Elastic waves in heterogeneous and porous media. Geophysics, 21:41-70.

Date of Initial Receipt: December 7, 1981 